\title{
Bimodal trait distributions with large variances question the reliability of trait-based aggregate models
}

\author{
Renato Mendes Coutinho ${ }^{1}$. Toni Klauschies ${ }^{2}$ - Ursula Gaedke ${ }^{2}$
}

Received: 22 September 2015 / Accepted: 21 February 2016 / Published online: 18 March 2016

(C) Springer Science+Business Media Dordrecht 2016

\begin{abstract}
Functionally diverse communities can adjust their species composition to altered environmental conditions, which may influence food web dynamics. Trait-based aggregate models cope with this complexity by ignoring details about species identities and focusing on their functional characteristics (traits). They describe the temporal changes of the aggregate properties of entire communities, including their total biomasses, mean trait values, and trait variances. The applicability of aggregate models depends on the validity of their underlying assumptions that trait distributions are normal and exhibit small variances. We investigated to what extent this can be expected to work by comparing an innovative model that accounts for the full trait distributions of predator and prey communities to a
\end{abstract}

Renato Mendes Coutinho and Toni Klauschies contributed equally to this work.

Electronic supplementary material The online version of this article (doi:10.1007/s12080-016-0297-9) contains supplementary material, which is available to authorized users.

Toni Klauschies

tklausch@uni-potsdam.de

Renato Mendes Coutinho

renatomc@ift.unesp.br

Ursula Gaedke

gaedke@uni-potsdam.de

1 Instituto de Física Teórica, Universidade Estadual Paulista Júlio de Mesquita Filho, São Paulo, Brazil

2 Department of Ecology and Ecosystem Modeling, Institute for Biochemistry and Biology, University of Potsdam, Potsdam, Germany corresponding aggregate model. We used a food web structure with well-established trade-offs among traits promoting mutual adjustments between prey edibility and predator selectivity in response to selection. We altered the shape of the trade-offs to compare the outcome of the two models under different selection regimes, leading to trait distributions increasingly deviating from normality. Their biomass and trait dynamics agreed very well for stabilizing selection and reasonably well for directional selection, under which different trait values are favored at different times. However, for disruptive selection, the results of the aggregate model strongly deviated from the full trait distribution model that showed bimodal trait distributions with large variances. Hence, the outcome of aggregate models is reliable under ideal conditions but has to be questioned when confronted with more complex selection regimes and trait distributions, which are commonly observed in nature.

Keywords Fitness gradient - Communities as complex adaptive systems $\cdot$ Moment closure for trait-based aggregate model approaches $\cdot$ Multimodal trait distributions - Lumpiness in pattern formation and self-organization - Shape of trade-offs and stabilizing and disruptive selection

\section{Introduction}

Diverse ecological communities can adjust their composition and corresponding (mean) properties to local environmental conditions, which may in turn alter their dynamics. To investigate this complex but important causal chain, ecologists have increasingly advocated trait based approaches (Leibold and Norberg 2004; Webb et al. 2010). They depict species by their functional traits and corresponding trait 
values. The heritable trait variation existing within and among species can be represented by a single continuous trait distribution that may change in response to natural selection. This change may proceed mainly through species sorting, as often assumed in discrete approximations of full trait distribution (FTD) models (Norberg et al. 2001; Bruggeman and Kooijman 2007; Merico et al. 2009). However, such multispecies models require a high number of species entities, i.e., state variables, to reduce potential artefacts that may arise from assigning a priori exact trait values to the species present. This increases model complexity and computational effort and may preclude a detailed understanding of the feedback between biomass and trait dynamics.

In order to turn intractable exact equations into tractable approximate equations, theoretical ecologists developed aggregate models. They describe the temporal changes of the aggregate properties of an entire community, such as its total biomass, mean trait value and trait variance, in response to selection. The mean trait value and trait variance correspond to the first and second central moments of the trait distribution and reflect the average strategy and the overall functional diversity of the community (Wirtz and Eckhardt 1996; Merico et al. 2009; Tirok et al. 2011) or functional group (Norberg et al. 2001; Terseleer et al. 2014). However, their temporal changes generally depend on higher-order moments, particularly the third and fourth central moment (related to skewness and kurtosis) of the trait distribution. These are usually neglected or estimated from lower-order moments by assuming trait values to be normally distributed and trait variances to be small (Wirtz and Eckhardt 1996; Merico et al. 2009; Tirok et al. 2011; Terseleer et al. 2014).

The assumption of such a narrow unimodal trait distribution is generally valid under stabilizing selection promoting the dominance of species with very similar trait values as seen in models (Merico et al. 2009) and observed, e.g., during blooms of cyanobacteria (Scheffer et al. 1997) or pennate diatoms (Horn et al. 2011) in freshwater phytoplankton. However, temporal changes in abiotic forcing (Fox et al. 2010; Sommer et al. 2012) or internal feedback mechanisms (e.g., biomass-trait feedback, Tirok and Gaedke 2010 or eco-evolutionary dynamics, Becks et al. 2010) may strongly alter the selection pressure on natural communities within ecological time, giving rise to directional or even disruptive selection. Under these conditions, the shape and variance of the trait distributions are expected to differ strongly from a narrow unimodal one, questioning the reliability of aggregate models. For example, under directional selection, trait distributions should exhibit skewness because different trait values are favored at different times, while disruptive selection will promote the co-occurrence of two greatly different strategies, giving rise to bimodal trait distributions with large variances. In such cases, species with trait values much distant from the current mean trait value are frequently abundant, so that the mean trait value does not represent the majority of the community. According to Jensen's inequality, the evaluation of functions at their mean trait value is not appropriate if the trait distributions exhibit large variances and if functional relationships are strongly nonlinear, as generally found in nature. Even when accounting for a second-order approximation, as it is done in aggregate models, the truncation of a Taylor expansion after the second term will strongly bias the results when the higherorder derivatives do not vanish and the trait variance is large.

We thus reevaluated the reliability of the aggregate model approach for different selection regimes. Following empirical evidence (Tirok and Gaedke 2007), we considered a predator-prey system in which prey species vary in their intrinsic growth rates and vulnerabilities to predation, while predator species differ in respect to their prey selectivity and ability to graze efficiently on low prey densities, and we assumed corresponding trade-offs between the traits. To account for the FTD of the prey and predator communities, we generalized a multispecies predator-prey model (Tirok and Gaedke 2010; Bauer et al. 2014) to one that considers a continuum of trait values. We then derived a corresponding aggregate model from the FTD model using a moment closure technique based on the assumption of normally distributed trait values. Finally, we compared the temporal dynamics and long-term behavior of the two models across a set of different trade-off shapes that correspond to different selection regimes. In contrast to multispecies and aggregate models, our FTD model does not make any assumptions about the exact trait values present and the shape of the trait distributions, which confers a great generality to this innovative approach.

We show that the aggregate model works well under strongly stabilizing selection but increasingly lacks accuracy when the selection regime changes towards more directional or even disruptive selection. It predicts under directional selection very similar temporal averages and types of dynamics of the biomasses and mean trait values, but accumulates large errors in the trait variances and timescale of the biomass-trait feedback. Under disruptive selection, the aggregate model entirely fails to match the results of the FTD model, showing very different temporal averages and dynamics of all aggregate properties of both communities. Indeed, the FTD model reveals strongly skewed or bimodal trait distributions with large variances, which the aggregate model is unable to accommodate, displaying very different alternative states. 


\section{Methods}

The first two subsections describe the two model approaches we are going to compare under different tradeoff shapes: a full trait distribution (FTD) model and a corresponding aggregate model version. In the third subsection, we explain how trends in the shape of the trade-off functions alter the selection regime from stabilizing, over directional, to disruptive selection. Finally, we outline the analyses performed.

\section{Full trait distribution model}

Building on previous work (Tirok and Gaedke 2010; Tirok et al. 2011; Bauer et al. 2014), we used a modification of the classical Rosenzweig-MacArthur predator-prey model (Rosenzweig and MacArthur 1963) to simulate a continuous set of predator and prey species differing in their selectivity $\omega$ and edibility $\phi$, respectively. Hence, the predators can be more or less selective and the prey can be more or less susceptible to predation. The functional traits of both trophic levels determine the grazing rate $g$ of each predator on each prey species and adhere to well-established trade-offs. The prey's edibility trades off with their intrinsic growth rate $r^{\prime}$, so that $r^{\prime}$ becomes higher the more edible a prey is. The predator's selectivity trades off with their halfsaturation constant $M$ so that a more selective predator has a lower half-saturation constant (for details see "Trade-offs and selection regimes" section). To merge intra- and interspecific trait variation, we generalized the multispecies predator-prey model previously used (Tirok and Gaedke 2010; Bauer et al. 2014) to one that considers a continuum of trait values. Our corresponding FTD model allows any possible community composition by tracking the biomass of every phenotype within a finite trait range. Hence, we do not make any assumptions in advance about the species' or genotypes' exact trait values, or the shape of the communities trait distributions.

The prey $(A)$ and predator $(C)$ community biomasses change according to the following set of equations:

$$
\begin{aligned}
& \frac{\partial A(\phi, t)}{\partial t}=(r(\phi)+B(\phi)) A-\overbrace{\int g(\phi, \omega) C d \omega}^{\text {total consumption by predators }}+I_{A} \\
& \frac{\partial C(\omega, t)}{\partial t}=(e \underbrace{\int g(\phi, \omega) d \phi}_{\text {total prey grazed }}-d+B(\omega)) C+I_{C}
\end{aligned}
$$

where $e$ is the conversion efficiency, $d$ the predator mortality rate, and $I_{A}$ and $I_{C}$ the prey and predator immigration rates, respectively, preventing complete exclusion of any single trait value in the FTD model. Since we consider predominantly predator-prey interactions in invertebrates like phytoplankton and zooplankton communities, which often show dormancy, immigration may not only account for external input of trait variation but also capture germination from seed banks and resting stages, which are ubiquitous mechanisms maintaining functional diversity. We assumed a common carrying capacity, $K$, for the prey community and a type II functional response (Holling 1965) for the predators. The growth rate $r$ for a single prey type and the grazing rate $g$ for a single predator type are given by:

$$
\begin{gathered}
r(\phi)=r^{\prime}(\phi)(1-\underbrace{\frac{P^{\text {any prey }}\left(\phi^{\prime}\right) d \phi^{\prime}}{K}}_{\text {interspecific competition }}) \\
g(\phi, \omega)=g_{m} \frac{q(\phi, \omega) A(\phi)}{M(\omega)+\underbrace{\int q\left(\phi^{\prime}, \omega\right) A\left(\phi^{\prime}\right) d \phi^{\prime}}_{\text {sum over all prey }}},
\end{gathered}
$$

where $r^{\prime}$ is the maximum intrinsic growth rate of the prey, and $M, q$, and $g_{m}$ are the half-saturation constant, grazing preference, and maximum grazing rate of the predators. The index $\phi^{\prime}$ denotes that the integration is done over all prey species, independent of $\phi$, that is, of the particular species for which the growth or grazing rate is calculated. The preference function $q$ increases with decreasing values of $\omega$ and increasing values of $\phi$ and is given by:

$q(\phi, \omega)=[1+\exp (-b(\phi-c \omega))]^{-1}$.

Hence, non-selective predators $(\omega \approx 0)$ have high $q$ values for all prey species, whereas more selective ones $(\omega \gg 0)$ have high $q$ values only for a more restricted prey spectrum $(\phi \gg 0)$, the range of which is quantified by the parameter $c$. The value of $b$ determines the sharpness of the transition of the $q$ values from non-preferred to preferred prey species. The boundary function $B$ in Eq. 1 accounts for all ecologically relevant processes, such as size and metabolic constraints, that restrict trait values to a biologically feasible range, which we take to be between $[0,1]$ since, e.g., prey species cannot be more than fully edible $(\phi=1)$ or less than entirely inedible $(\phi=0)$. The boundary function $B$ is modeled as:

$B(x)=-(\exp (-w x)+\exp (w(x-1)))$,

where $x$ refers to the edibility or selectivity of the respective prey or predator species. The parameter $w$ determines the overall shape of $B$ and was set to 40 so that $B$ is very close to zero over most of the trait range but gets 
increasingly negative close to the minimum $(x=0)$ and the maximum $(x=1)$ trait values, making trait values at the extremes unfavorable. The boundary function $B$ prevents the FTD model to exhibit any edge effects such as non-smooth trait distributions, which would arise if we simply restrict the feasible trait range, and it keeps the FTD model and corresponding aggregate model fully comparable. The implementation of a boundary function is akin to methods used in evolutionary biology where general fitness functions are implemented that give rise to overall stabilizing selection restricting standing trait variation (Doebeli et al. 2007; Baptestini et al. 2009) and changes in mean trait values (Saloniemi 1993; Abrams 2006) of populations or communities to their ecologically feasible ranges.

In building this model, we assume a strictly trait-based approach, where organisms are characterized exclusively by their trait values and all individuals produce only new individuals with exactly the same trait value. This is in line with communities predominantly composed by asexually reproducing unicellular organisms or small metazoans, dominating marine and freshwater ecosystems (Sommer et al. 2012). Hence, we adopted parameter values that are typical of a system consisting of phytoplankton and ciliates (protozoans; cf. Tirok and Gaedke 2007), in which cell sizes increase with trophic level and so, by allometry, weight-specific rates decrease with trophic level (Tang 1995; Hansen et al. 1997). For a detailed description of the parameters and corresponding values used, see Table 1.

At every moment in time, the FTD model yields the biomass densities of the prey and predator communities as a function of their respective traits. In order to describe general changes in the magnitude, location, and shape of these trait distributions, we consider the three aggregate properties of those communities: total biomasses, mean trait values, and trait variances, defined by:

$$
\begin{aligned}
A_{T} & =\int A(\phi) d \phi \\
\bar{\phi} & =\frac{1}{A_{T}} \int \phi A(\phi) d \phi \\
v_{\phi} & =\frac{1}{A_{T}} \int(\phi-\bar{\phi})^{2} A(\phi) d \phi .
\end{aligned}
$$

with analogous expressions for predator community's quantities $\left(C_{T}, \bar{\omega}\right.$, and $\left.v_{\omega}\right)$. The mean trait values may reflect the most abundant species in the prey and predator communities, whereas the trait variances denote their functional diversities.

\section{Aggregate model}

The FTD model describes the temporal changes in the biomass density of each trait value, from which the aggregate properties of the prey and predator communities are calculated. Alternatively, one may focus directly on the temporal changes of the aggregate properties using moment approximation methods. In this case, the dynamics of the FTD model is approximated by a corresponding aggregate model which tracks only the changes in the total biomasses $\left(A_{T}\right.$ and $\left.C_{T}\right)$, mean trait values $(\bar{\phi}$ and $\bar{\omega})$, and trait variances $\left(v_{\phi}\right.$ and $\left.v_{\omega}\right)$ of the prey and predator communities. Following previous studies (Norberg et al. 2001; Savage et al. 2007), we formulated an aggregate model that can be derived through a moment approximation, that truncates the moment expansion of the trait distribution at the second order, assuming trait variances to be small or higherorder derivatives of the species' fitness, i.e., of its per-capita net growth rate, to vanish (for a detailed derivation see "Appendix A: Derivation of the aggregate model"). In
Table 1 Description and values of parameters used

\begin{tabular}{llll}
\hline Symbol & Description & Value & Unit \\
\hline$K$ & Prey carrying capacity & 10 & $\mathrm{~g} \mathrm{Cm}^{-2}$ \\
$\bar{r}$ & Mean growth rate of prey & $9 / 8$ & $\mathrm{day}^{-1}$ \\
$m_{r}$ & Ratio between maximum and minimum growth rate & 8 & - \\
$d$ & Predator mortality rate & 0.15 & $\mathrm{day}^{-1}$ \\
$e$ & Predator growth efficiency & 0.2 & - \\
$g_{m}$ & Maximum grazing rate & 2 & $\mathrm{day}^{-1}$ \\
$m_{M}$ & Ratio between minimum and maximum half-saturation constant & $1 / 8$ & - \\
\hline$M^{-1}$ & Mean grazing rate of predators at low prey densities & $\frac{\ln (8)}{7}$ & $\mathrm{~g} \mathrm{Cm}^{-2}$ \\
$b$ & Trade-off exponent for sharpness of the predator preference $q$ & 6 & - \\
$c$ & Trade-off coefficient relating $\phi$ and $\omega$ in the preference $q$ & 0.9 & - \\
$I_{A}$ & Prey immigration rate & 0.0001 & $\mathrm{~g} \mathrm{Cm}^{-2} \mathrm{day}^{-1}$ \\
$I_{C}$ & Predator immigration rate & 0.00002 & $\mathrm{~g} \mathrm{Cm}^{-2} \mathrm{day}^{-1}$ \\
$w$ & Steepness of the boundary function & 40 & - \\
\hline
\end{tabular}


order to derive self-contained expressions for the temporal development of the aggregate properties, we applied a moment closure which assumes trait values to be normally distributed (cf. Wirtz and Eckhardt 1996; Merico et al. 2009; Tirok et al. 2011). The aggregate properties change according to the following set of equations:

$$
\begin{aligned}
\frac{d A_{T}}{d t} & =A_{T}\left(R_{A}(\bar{\phi})+\left.\frac{v_{\phi}}{2} \frac{d^{2} R_{A}}{d \phi^{2}}\right|_{\phi=\bar{\phi}}\right)+I_{A} \\
\frac{d C_{T}}{d t} & =C_{T}\left(R_{C}(\bar{\omega})+\left.\frac{v_{\omega}}{2} \frac{d^{2} R_{C}}{d \omega^{2}}\right|_{\omega=\bar{\omega}}\right)+I_{C} \\
\frac{d \bar{\phi}}{d t} & =\left.v_{\phi} \frac{d R_{A}}{d \phi}\right|_{\phi=\bar{\phi}}+\frac{I_{A}}{A_{T}}\left(\frac{1}{2}-\bar{\phi}\right) \\
\frac{d \bar{\omega}}{d t} & =\left.v_{\omega} \frac{d R_{C}}{d \omega}\right|_{\omega=\bar{\omega}}+\frac{I_{C}}{C_{T}}\left(\frac{1}{2}-\bar{\omega}\right) \\
\frac{d v_{\phi}}{d t} & =\left.v_{\phi}^{2} \frac{d^{2} R_{A}}{d \phi^{2}}\right|_{\phi=\bar{\phi}}+\frac{I_{A}}{A_{T}}\left[\frac{1}{12}-v_{\phi}+\left(\frac{1}{2}-\bar{\phi}\right)^{2}\right] \\
\frac{d v_{\omega}}{d t} & =\left.v_{\omega}^{2} \frac{d^{2} R_{C}}{d \omega^{2}}\right|_{\omega=\bar{\omega}}+\frac{I_{C}}{C_{T}}\left[\frac{1}{12}-v_{\omega}+\left(\frac{1}{2}-\bar{\omega}\right)^{2}\right],
\end{aligned}
$$

where $R_{A}$ and $R_{C}$ are the instantaneous per capita net growth rates of the prey and the predators evaluated at their respective mean trait values $\bar{\phi}$ and $\bar{\omega}$ :

$$
\begin{aligned}
& R_{A}(\phi)=r(\phi)-C_{T}\left(G(\phi, \bar{\omega})+\left.\frac{v_{\omega}}{2} \frac{\partial^{2} G}{\partial \omega^{2}}\right|_{\omega=\bar{\omega}}\right)+B(\phi) \\
& R_{C}(\omega)=e A_{T}\left(G(\bar{\phi}, \omega)+\left.\frac{v_{\phi}}{2} \frac{\partial^{2} G}{\partial \phi^{2}}\right|_{\phi=\bar{\phi}}\right)-d+B(\omega), \quad(7)
\end{aligned}
$$

and $G$ is the aggregate grazing function, defined below.

Changes in the prey and predator community biomasses follow the same functional forms as in the FTD model,

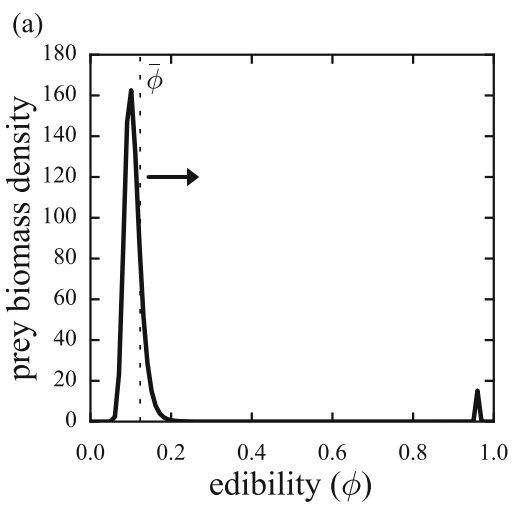

Fig. 1 Difference between the FTD and aggregate model approach. a Biomass density distribution with respect to the edibility $(\phi)$ of the prey at a certain moment in time. b Corresponding per capita net growth rate as a function of the trait $\phi$ (fitness landscape), calculated using the FTD model. The vertical dashed line represents the mean trait value and the arrow indicates the direction of its change. In the aggregate model, the rate of change of the mean trait is proportional to while changes in the mean traits and trait variances are determined, respectively, by the first and second derivatives of the per capita net growth rates. Hence, the mean trait values change with a speed proportional to the local fitness gradient, i.e., the first derivative of the per-capita net-growth rate evaluated at the respective mean trait value, and to the trait variance. Furthermore, Eq. 6 includes additional terms to account for non-linear averaging and changes in the location and width of the assumed normal trait distributions due to immigration (cf. "Appendix A: Derivation of the aggregate model" and Norberg et al. 2001). In accordance with the FTD model, the immigration rates $I_{A}$ and $I_{C}$ preclude the trait variance from becoming exactly zero in the aggregate model, whereas the boundary function $B$ constrains changes in the mean trait values $\bar{\phi}$ and $\bar{\omega}$ to the delimited range and ensures that the trait variance vanishes when the mean trait value approaches an extreme (4). The growth and grazing functions of the aggregate model are given by

$$
\begin{aligned}
r(\phi) & =r^{\prime}(\phi)\left(1-\frac{A_{T}}{K}\right) \\
G(\phi, \omega) & =\frac{g_{m} q(\phi, \omega)}{M(\omega)+\left(q(\bar{\phi}, \omega)+\left.\frac{v_{\phi}}{2} \frac{\partial^{2} q(\phi, \omega)}{\partial \phi^{2}}\right|_{\phi=\bar{\phi}}\right) A_{T}} .
\end{aligned}
$$

This model represents the closest aggregate model one can build using the same assumptions as in the FTD model. The two model approaches fundamentally differ in the way the aggregate properties of the trait distribution change. In the aggregate model, the changes are consequences only of the fitness landscape, i.e. the per-capita net-growth rate as a function of the respective trait, around the current mean trait value, whereas the FTD model accounts for the entire fitness landscape. The fitness landscape at a given point in time can

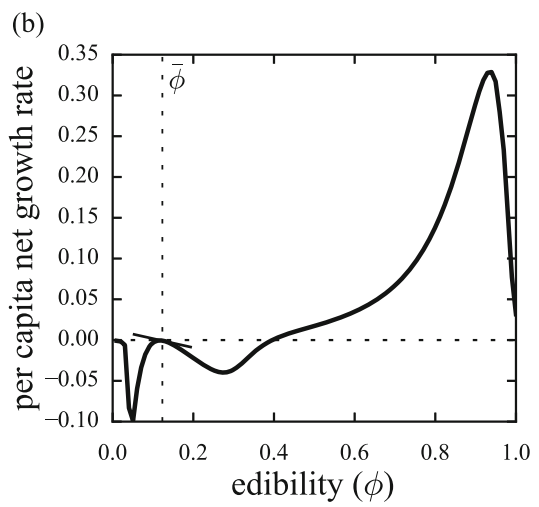

the value of the fitness gradient at the mean trait value (depicted by the slope of the tangent black line), while for the FTD model the contributions of all trait values are summed over. This implies in the present example that the temporal changes in the mean trait value strongly differ in magnitude and even direction due to the high growth rates at high values of $\phi$ 
be rather complex due to the non-linearity usually inherent to the feeding relationships and the trade-offs underlying the system. As a result, in the FTD model, the mean trait value may change in the opposite direction of the gradient of the fitness landscape evaluated at the mean trait value (Fig. 1).

\section{Trade-offs and selection regimes}

Species can use resources either to increase reproduction or to counteract stress, like grazing losses (Strauss et al. 2002; Norberg 2004). For this reason, we assume the maximum growth rate of the prey to increase with its edibility, so that fast growing prey species are highly vulnerable to grazing and vice versa. In addition, the predators' capacity to exploit many different food items, i.e., its selectivity, may trade off with the ability to exploit low prey densities (cf. Straub et al. 2011). Hence, we assume the half-saturation constant of the predators to attain its maximum for the least selective species, for which $M=M_{\max }$, while the most selective species has a half saturation constant $m_{M}$ times smaller. The explicit expressions of the trade-offs are given by the following set of equations:

$$
\begin{aligned}
r^{\prime}(\phi) & =r_{\min }(\beta)\left(1+\left(m_{r}-1\right) \phi^{\beta}\right) \\
M(\omega) & =M_{\max }(\beta)\left(1+\left(m_{M}-1\right) \omega^{\beta}\right),
\end{aligned}
$$

where $\beta$ determines the shape of the trade-offs, which is linear when $\beta=1$ (Fig. 2a-c).

We systematically altered the shape of the trade-offs to manipulate the selection regime on the prey and predator communities (cf. Levins 1962; Abrams 2006). When $\beta$ is small, $r^{\prime}$ increases quickly for small $\phi$, but slowly for high $\phi$, and $M$ declines steeply at low $\omega$, but gently for high $\omega$ (Fig. 2a). This gives rise to stabilizing selection at both trophic levels, in which intermediate values of both $\phi$ and $\omega$ are favored, since they experience relatively low costs for their relatively high growth and grazing rates. For large values of $\beta$, the selection pressure is reversed because $r^{\prime}$ increases slowly at low $\phi$ and quickly at high $\phi$, while $M$ decreases slowly at low, but steeply for high $\omega$ (Fig. 2c), thus favoring both extreme trait values in both trophic levels. This constitutes a case of disruptive selection, in which intermediate trait values are maladapted, since they experience relatively high costs for their relatively low growth and grazing rates. Intermediate values of $\beta$ provoke a balance between costs and benefits along the trait range, where each species may be favored at a given moment in time depending on the current prey and predator biomasses, resulting in recurrent changes in the dominant trait value and thus directional selection (Fig. 2b). Hence, increasing $\beta$ from low to high values changes the selection regime on the prey and predator communities from stabilizing to disruptive selection, thereby altering the expected shape of the trait distributions (Fig. 2).

We can depict the expected long-term effect of the changes in $\beta$ on the shape of the trait distributions by looking at the effective carrying capacity of the rate of change of the prey biomasses. The latter can be expressed as (cf. Eqs. 1 and 2):

$$
\frac{\partial A(\phi, t)}{\partial t}=\left[\left(r^{\prime}(\phi)-\eta(\phi)+B(\phi)\right)\left(1-\frac{A_{T}}{K^{\prime}(\phi)}\right)\right] A+I_{A},
$$

where $K^{\prime}(\phi)=K\left(1+\frac{B(\phi)-\eta(\phi)}{r^{\prime}(\phi)}\right)$ denotes the effective carrying capacity of the prey, which accounts for the effects of both the per capita death rate by predation $\eta(\phi)=$ $g_{m} \int \frac{q(\phi, \omega) C(\omega)}{M(\omega)+\int q\left(\phi^{\prime}, \omega\right) A\left(\phi^{\prime}\right) d \phi^{\prime}} d \omega$ and the intrinsic growth rate $r^{\prime}(\phi)$. The functions $\eta$ and $r^{\prime}$ are monotonically increasing
Fig. 2 The top panels show the trade-off relations between the maximum growth rate $\left(r^{\prime}\right.$, green solid line $)$ and edibility $(\phi)$, and between the grazing half-saturation constant ( $M$, blue dashed line) and selectivity $(\omega)$ for three values of $\beta$. The arrows in panels $\mathbf{a}$ and $\mathbf{c}$ indicate the direction of selection on traits, in comparison to the linear case. The bottom panels show how the shape of the trade-offs shapes the effective carrying capacity $K^{\prime}$ of prey, which can be thought of as an expected trait distribution. It was calculated assuming a total biomass of $K / 2$ for prey and $K / 3$ for predators, with uniform trait distributions at both trophic levels

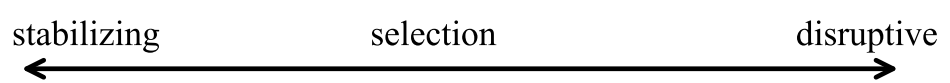

(a) $\beta=0.2$

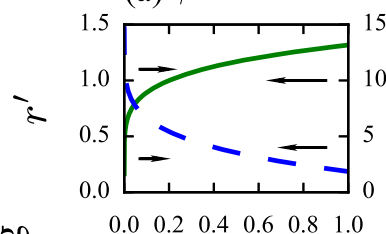

(d)

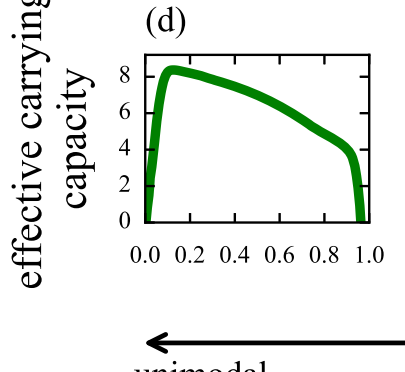

unimodal (b) $\beta=1.0$

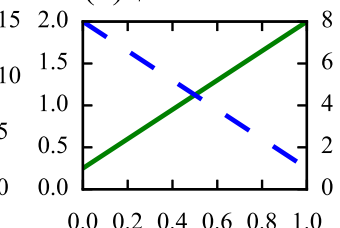

(e)

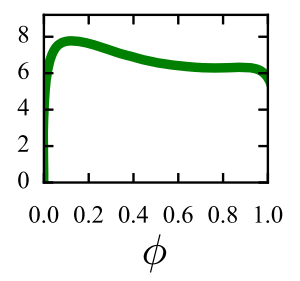

trait distributions (c) $\beta=5.0$

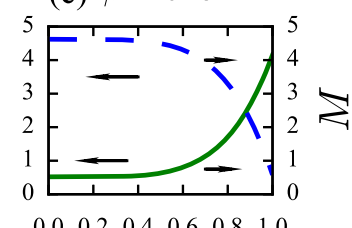

(f)

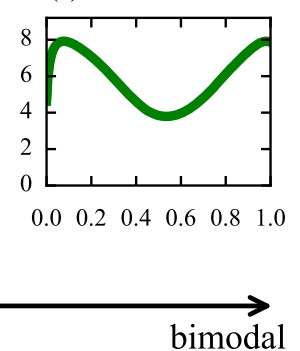


in $\phi$, but only $r^{\prime}$ changes with $\beta$. This formulation makes explicit the relative roles of the intrinsic growth rate and predation for overall prey competition, i.e., $K^{\prime}$, and shows that the long-term trait distribution of the prey is expected to be strongly concentrated when $\beta$ is low (Fig. 2d), whereas it should present peaks at both low and high trait values for large $\beta$, giving rise to large trait variances (Fig. 2f). In addition, we expect the trait distributions to be on average rather uniform for $\beta \approx 1$ (Fig. 2e).

To avoid any confounding effects when changing the parameter $\beta$, we maintained the relative growth and grazing rates, i.e., the ratios between the maximum and minimum growth rates $\left(m_{r}\right)$ and half-saturation constants $\left(m_{M}\right)$, constant within each trophic level. In addition, we chose the dependence of $r_{\min }$ and $M_{\max }$ on $\beta$ in such a way that, given a homogeneous distribution of traits, the averages of the prey's maximum growth rate, $\bar{r}=\int_{0}^{1} r^{\prime}(\phi) d \phi$, and the predator's grazing rate at low prey densities, $\overline{\left(\frac{g_{m}}{M}\right)}=$ $\int_{0}^{1} \frac{g_{m}}{M(\omega)} d \omega$, were constant. This is important because, otherwise, the direct increase or decrease in prey productivity or in sensitivity of predators to the amount of prey would shadow any effect of changes in $\beta$. These assumptions lead to:

$$
\begin{aligned}
r_{\text {min }}(\beta) & =\bar{r}\left(1+\frac{(m r-1)}{1+\beta}\right)^{-1} \\
M_{\text {max }}(\beta) & =\frac{{ }_{2} F_{1}\left(1, \frac{1}{\beta}, 1+\frac{1}{\beta}, m_{M}\right)}{\overline{M^{-1}}},
\end{aligned}
$$

where ${ }_{2} F_{1}$ is the hypergeometric function.

\section{Numerical simulations and data analysis}

We evaluated the suitability of the aggregate model under different selection regimes using numerical simulations for different values of $\beta$. As initial conditions, we assumed total biomasses in each trophic level to be $1 \mathrm{~g} \mathrm{Cm}^{-2}$ (cf. Gaedke and Wickham 2004) and both traits to be normally distributed with means of 0.5 or 0.9 and variances of $1 / 400$. These two initial conditions led to different long-term solutions, so we explored the extent of these bistable regions and how they change with $\beta$. We continued solutions in these regions using the final state of a simulation as initial condition for the next one with a slightly modified value of $\beta$, hence revealing the extent of the range of values of $\beta$ where bistability is present. The FTD model and its aggregate properties were numerically integrated using a discretization along the trait axes $(n=100)$.

We evaluated the agreement between the two model approaches by comparing the temporal dynamics and longterm behavior of their aggregate properties as a function of $\beta$. The latter was done by calculating the temporal average and variation of the community biomasses, the mean trait values, and the trait variances. We also evaluated the stability of the predator-prey dynamics by calculating the coefficient of variation (CV) for both prey and predator community biomasses. In order to better understand potential differences between the two model approaches, we also looked at the trait distributions' skewness $(S)$ and (excess) kurtosis $(\kappa)$, obtained from solutions of the FTD model, which are directly related to the third and fourth order central moments. In general, large absolute values of skewness or kurtosis imply that the shape of the trait distribution strongly deviates from a normal one, highlighting the importance of higher-order moments.

A small absolute skewness may indicate that the trait distribution is rather symmetrical, whereas a large absolute skewness points to a trait distribution possessing a heavy tail on the right (positive skewness) or left (negative skewness) side of the distribution's mean trait value. Since we are mainly interested in the overall magnitude of a trait distribution's skewness, rather than its sign, we computed the average of the skewness' absolute values over a long time.

For symmetrical trait distributions, large negative values of kurtosis $(-2<\kappa \ll 0)$ indicate light tails and flatness, as seen for uniform and, in its extreme, bimodal trait distributions, which have most density at the distribution's shoulders, i.e., around $\pm 1 S D$ of the mean (DeCarlo 1997). In contrast, large positive values of kurtosis $(\kappa \gg$ $0)$ point toward peaked and heavy-tailed trait distributions, since this describes the dispersion of density around $\pm 1 S D$ of the mean (Moors 1986; DeCarlo 1997). High skewness, however, strongly affects kurtosis values, and the relationship between these measures is hard to disentangle (Blest 2003; Jones et al. 2011). Therefore, we use a measure for bimodality based on Pearson's inequality (Pearson 1929):

$P(t)=S^{2}(t)-\kappa(t)$.

The value of $P$ is maximal for the two-point binomial $(P=$ 2), amounts to 1.2 for a uniform distribution, and to 0 for a normal distribution (Klaassen et al. 2000). We record how frequently $P$ surpasses the value of 1.2 over a long time as evidence for bimodal trait distributions.

Simulations and analyses of the aggregate model were performed in MATLAB, version 7.13, using solver ode23 for ODEs (The MathWorks Inc., Natick, MA, 2011). We increased the precision of the solver by setting the absolute and relative tolerance to $10^{-9}$ and $10^{-12}$ and the maximum step size to 0.1. The FTD model was implemented discretizing the trait axes and solving the resulting system of ODEs using the Python wrapper odeint provided by SciPy (Jones et al. 2001) for the LSODA solver contained in the library ODEPACK (Hindmarsh 1983). The simulation results are robust to the choice of solver algorithm. 


\section{Results}

We tested the performance of the aggregate model under different selection regime using the full trait distribution (FTD) model as benchmark. For trade-off shapes arising from values of $\beta$ below 0.45 , selection is strongly stabilizing as indicated by unimodal trait distributions with small trait variances within both prey and predator communities in the FTD model (Figs. 3, 4, and cf. Fig. 2d). As expected under such conditions, the two models predict very similar dynamics and long-term states of the aggregate properties, in which both edibility and selectivity settle at low values. The latter are linked to relatively low growth and grazing rates (Fig. 2) promoting stable biomass dynamics (Fig. 4). The remaining small differences between the two model approaches arise from the fact that the trait distributions in the FTD model are highly skewed and peaked despite being unimodal (Table 2). However, skewness and peakedness hardly influence the overall model predictions because of the low trait variances $(v \ll 1)$, implying even lower higher-order moments.

Decreasing the strength of stabilizing selection $(\beta>$ 0.45 ) allows prey species with very different trait values to increase their biomass share in the community of residents, leading to bimodal trait distributions with high standing variances in the FTD model (Fig. 3). The outcome of this is an increase in the mean trait value and thus in productivity of the prey community, causing lower and higher equilibrium biomasses of the prey and predator communities, respectively. In contrast to the FTD model, the aggregate model predicts much smaller changes with $\beta$ in the aggregate properties of the prey and predator communities. This occurs because to achieve a drastic change in the mean trait value, it is not sufficient that species with extreme trait values are able to increase their share of the community biomass. Rather, it is necessary that species with trait values close to the previously favored one become sufficiently fit to outcompete the resident species.
Fig. 3 Temporal averages (lines) and variation $( \pm 1$ standard deviation; shades) of total biomass, mean trait, and standing trait variance of the solutions of the FTD (red) and aggregate (green) models as the shape of the trade-offs $(\beta)$ is varied. All other parameters as in Table 1 . The dotted lines correspond to alternative long-term solutions, obtained by exploring different initial conditions, leading to distinct basins of attraction

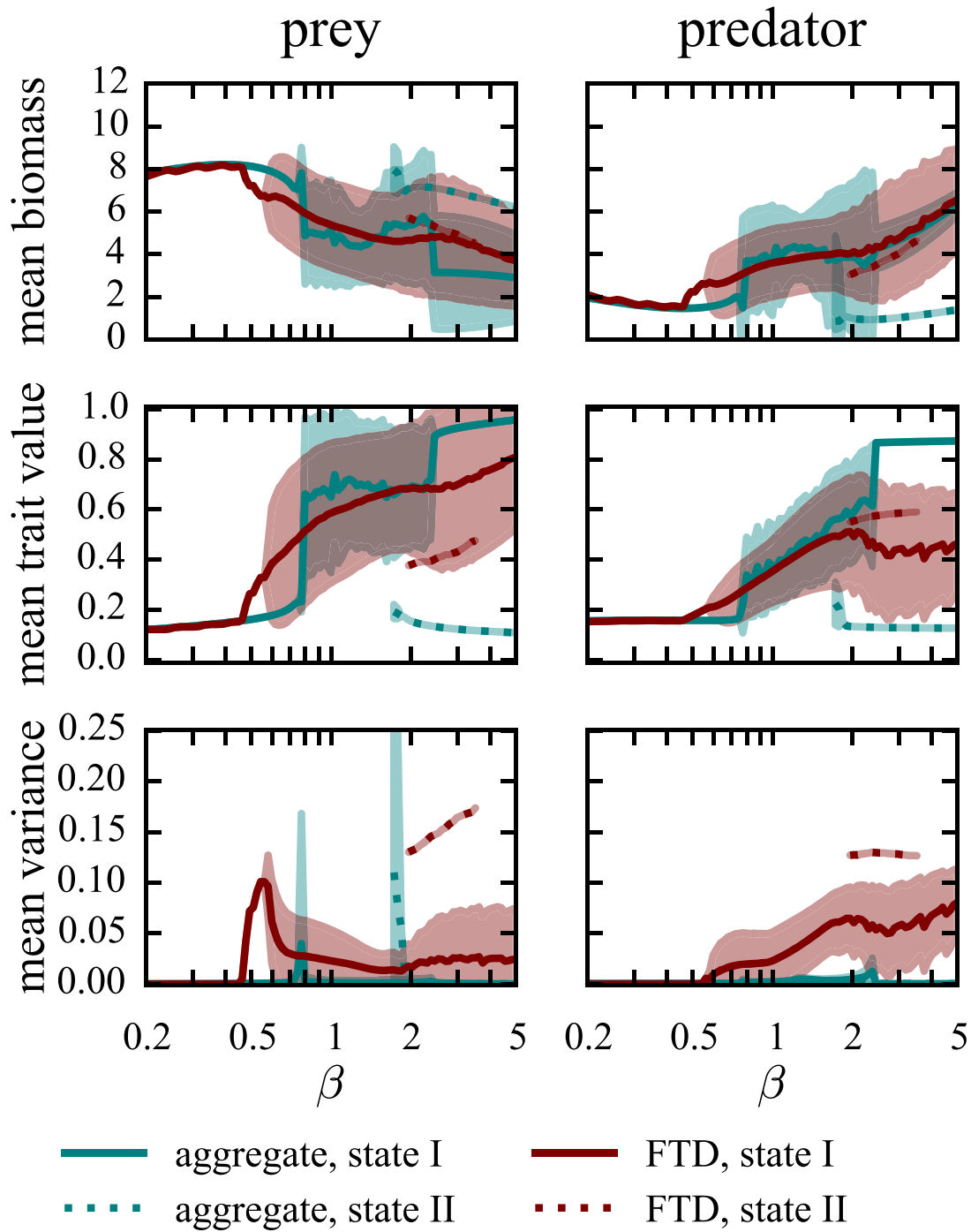


Fig. 4 Long-term solutions show stasis in the biomass and trait dynamics of the prey and predator communities under stabilizing selection $(\beta=0.2)$. Top: Total biomass of prey $A_{T}$ (green) and predators $C_{T}$ (blue) for the FTD (left) and aggregate (right) models. The panels just below show the mean trait values and trait variances of the trait distributions of prey edibility, $\phi$, and of predator selectivity, $\omega$, as a function of time. Bottom: The panel shows the final trait distribution of prey (biomass density as a function of edibility, $\phi$, in green), and predators (biomass density as a function of selectivity, $\omega$, in blue) in the FTD model
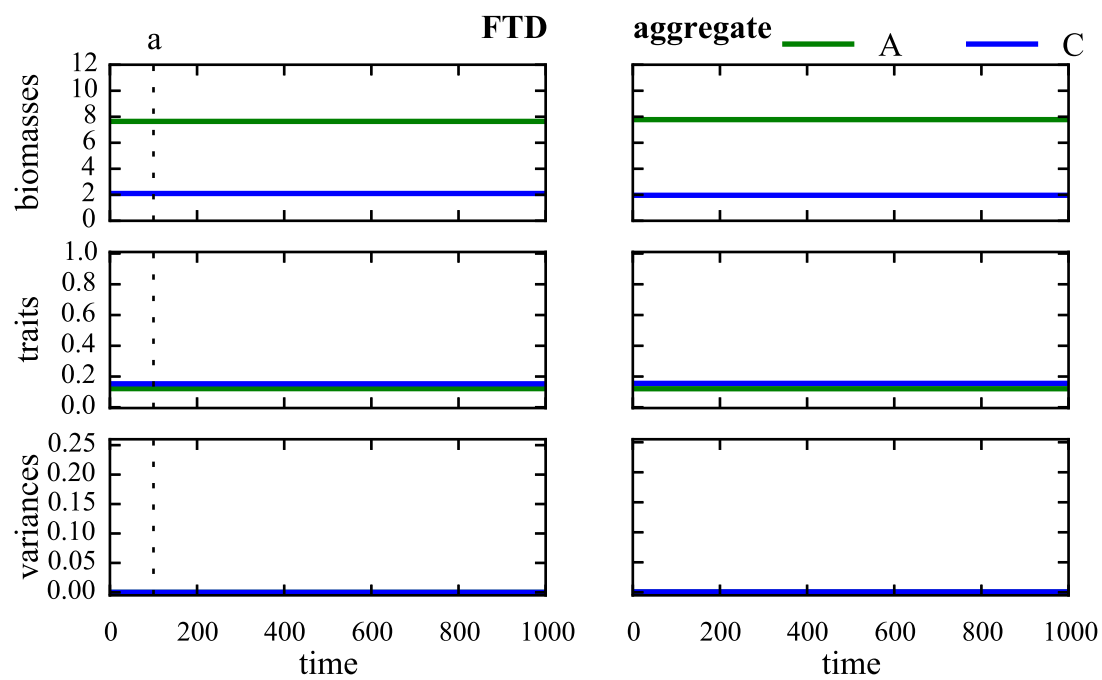

Trait distribution

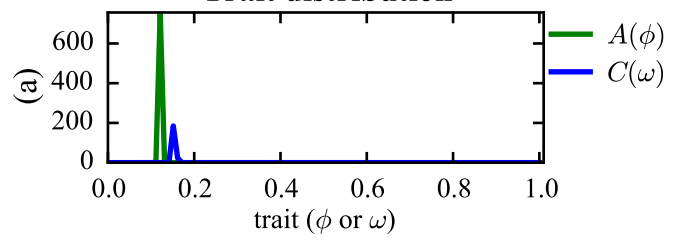

Higher values of $\beta$ (> 0.55) allow fast-growing well edible prey and selective predator species to recurrently increase their share. The relatively high growth and grazing rates (Fig. 2) strongly destabilize the biomass dynamics of the prey and predator communities in both models, giving rise to directional selection, in which different trait values are favored at different times. As a result, both models show considerable variation in the aggregate properties of the prey and predator communities over time (Figs. 3 and 5). However, the transition from stable to unstable dynamics occurs in the FTD model at a lower value of $\beta$ than in the aggregate model, producing substantial differences in their predictions of the temporal averages of the aggregate properties for values of $\beta$ between 0.55 and 0.75 . The higher stability of the aggregate model is due to the way the fitness landscape changes: the equilibrium trait value of the prey community established for $\beta<0.45$ is no longer a global maximum, but still remains a local maximum of the fitness landscape. This prevents the prey community from adapting its mean trait value to the currently favored one in the aggregate model. In contrast, in the FTD model, as well as in nature, it is sufficient that another species exists that is currently more fit and thus able to increase its share to the total biomass of the community, even when its trait value is very distant from the previously favored one.

Under directional selection, the environmental conditions change sufficiently fast to promote the emergence of skewed trait distributions with substantial variances in the FTD model (Fig. 5c). Since the direction of selection is changing over time, the trait distribution also changes its skewness between positive and negative values, passing through states in which it exhibits bimodality and a large variance (see $P$ in Table 2; Fig. 5b, d). In contrast to the effect of disruptive selection, these trait distributions are a combined result of past and present growth and death processes that exist only temporarily. In comparison to the FTD model, the aggregate model presents much lower trait variances (Fig. 3), since it is unable to accommodate the simultaneous occurrence of only lower and higher trait values, i.e., bimodal trait distributions. In the aggregate model, the mean trait value exhibits a period length that is more than two orders of magnitude larger than those in the FTD model, since its rate of change is proportional to the trait variance.

Despite this major deviation in the period length of the trait and biomass dynamics, both models show up to $\beta \approx 1.8$ very similar temporal averages of the biomass and mean trait values (Figs. 3 and 5). The aggregate model also depicts correctly the (mutual) trait adjustments of the prey and predator communities in response to altered selection pressures: in general, when highly edible (large $\phi$ ) species dominate the prey community, the predator community changes its composition toward more selective species (large $\omega$ ) that exploit the available prey more efficiently (Fig. 5a, b). Subsequently, the prey community escapes the enhanced predation pressure by changing its composition toward less edible species. Since the predators are no 
Table 2 Long-term behavior of the aggregate properties of the FTD (light gray) and the aggregate (white) models for both trophic levels (TL) under four distinct selection regimes (4 values of $\beta$ )

\begin{tabular}{|c|c|c|c|c|c|c|c|}
\hline$\beta$ & $\mathrm{TL}$ & model & $\mathrm{CV}$ & trait & $v$ & $|S|$ & $P$ \\
\hline \multirow[t]{4}{*}{0.2} & \multirow[t]{2}{*}{ prey } & cont. & $6 \times 10^{-5}$ & 0.12 & 0.0002 & 133 & $0 \%$ \\
\hline & & agg. & $5 \times 10^{-16}$ & 0.12 & 0.0004 & & \\
\hline & \multirow[t]{2}{*}{ predator } & cont. & $3 \times 10^{-5}$ & 0.15 & 0.0003 & 54 & $0 \%$ \\
\hline & & agg. & $7 \times 10^{-16}$ & 0.16 & 0.0007 & & \\
\hline \multirow[t]{4}{*}{1.0} & \multirow[t]{2}{*}{ prey } & cont. & 0.4 & 0.58 & 0.023 & 6.1 & $24 \%$ \\
\hline & & agg. & 0.5 & 0.65 & 0.0017 & & \\
\hline & \multirow[t]{2}{*}{ predator } & cont. & 0.4 & 0.35 & 0.023 & 4.6 & $40 \%$ \\
\hline & & agg. & 0.5 & 0.38 & 0.0021 & & \\
\hline \multirow[t]{4}{*}{3.0 (II) } & \multirow[t]{2}{*}{ prey } & cont. & $10^{-5}$ & 0.43 & 0.16 & 0.4 & $100 \%$ \\
\hline & & agg. & $5 \times 10^{-16}$ & 0.12 & 0.0005 & & \\
\hline & \multirow[t]{2}{*}{ predator } & cont. & $6 \times 10^{-5}$ & 0.58 & 0.13 & 0.4 & $100 \%$ \\
\hline & & agg. & $5 \times 10^{-16}$ & 0.13 & 0.0006 & & \\
\hline \multirow[t]{4}{*}{$5.0(\mathrm{I})$} & \multirow[t]{2}{*}{ prey } & cont. & 0.6 & 0.81 & 0.024 & 86 & $29 \%$ \\
\hline & & agg. & 0.7 & 0.96 & 0.0003 & & \\
\hline & \multirow[t]{2}{*}{ predator } & cont. & 0.4 & 0.46 & 0.08 & 1.6 & $100 \%$ \\
\hline & & agg. & 0.2 & 0.87 & 0.0003 & & \\
\hline
\end{tabular}

The measures correspond to the numerical solutions shown in Figs. 4, 5, 6 (state I), and 7 (state II). We show the coefficient of variation of the community biomasses (CV) and the long-term temporal averages of the mean trait value, trait variance $(v)$, and absolute skewness $(|S|)$. We also present the proportion of time when the trait distributions were bimodal according to Pearson's measure of bimodality $(P$; see methods, "Numerical simulations and data analysis"). The last two measures apply only to the FTD model

longer able to control the dominant species, the prey community biomass increases, whereas the predator community biomass decreases (Fig. 5b). The predator community responds to the altered selection pressure by decreasing its selectivity (Fig. 5c). The enhanced grazing pressure on the prey community dampens both, the increase in prey biomass and the decrease in predator biomass. However, the nonselective predators grow very slowly and therefore need a long time to recover. After building up sufficiently high biomasses, the predators are finally able to control the less edible prey. This, in turn, results in a shift within the prey composition toward more edible species, since they are able to outcompete the less edible ones due to their higher growth rates (Fig. 5d). From then onwards, the pattern repeats. As a result of this biomass-trait feedback, both models predict out-of-phase dynamics between prey and predator community biomasses. However, the high similarity of the predictions from the two models under directional selection strongly rely on immigration ("Appendix B: Model version with mutation"). When maintaining the trait variance by trait diffusion instead of immigration, the aggregate model results no longer match the results of the corresponding FTD model.

Increasing $\beta$ to values above 1.8 changes the selection regime toward more disruptive selection, in which the two model approaches substantially differ in their predictions about the temporal dynamics and long-term states of the aggregate properties (Fig. 3). Although both models exhibit bistability, the parameter range is much larger for the aggregate model. In one state ("state I"), the aggregate model features unstable predator-prey dynamics in which the prey and predator communities exhibit intermediate to high values of edibility and selectivity, respectively, which is in line with our findings from the FTD model for values of $\beta$ up to 2.4. However, for higher values of $\beta$, the two model approaches strongly differ in their predictions about the temporal dynamics and long-term states of the aggregate properties. The FTD model shows complex predator-prey cycles where high-frequency biomass oscillations are superimposed upon low-frequency trait oscillations (Fig. 6; for an animation of the temporal dynamics see also Online Resource 1). The prey and predators exhibit phase relationships of a quarter-period at the high-frequency and of a half-period at the low-frequency oscillations. In addition, the trait distributions of the prey and predator communities often considerably deviate from a normal distribution, displaying recurrently very large variances and skewnesses as well as bimodality most of the time (Fig. 6a-d, Table 2). As a result, the effects of typical non-linearities enclosed in the grazing structure on the temporal development of the lowerorder moments (i.e., aggregate properties) are enhanced by large higher-order moments. Because of this, the aggregate model here strongly deviates from the FTD model, predicting regular predator-prey cycles instead of out-ofphase dynamics, with constantly highly edible prey being grazed upon by highly selective predators. The latter results from the fact that the mean trait values of the prey and predator communities hardly change over time in the aggregate model because they are constrained by local fitness maximization.

In the alternative state ("state II"), the aggregate model and the FTD model both achieve stasis in the biomass and trait dynamics (Fig. 7). However, the aggregate model entirely fails to match the predictions of the FTD model as it features low biomasses of non-selective predators grazing on high biomasses of low edibility prey. In contrast, the FTD model predicts bimodal trait distributions within both the prey and predator communities, where both low and high edibility prey, and both non-selective and highly selective predator species coexist (Fig. 7, Table 2). This results in prey and predator biomasses which are strongly overand underestimated by the aggregate model, respectively. While this basin of attraction ("state II"; Fig. 3) vanishes in the FTD model when increasing $\beta$ to values above 3.5 , it 
Fig. 5 Long-term solutions show recurrent trait shifts in both prey and predator communities under directional selection $(\beta=1)$. Top: Total biomass of prey $A_{T}$ (green) and predators $C_{T}$ (blue) for the FTD (left) and aggregate (right) model. The panels just below show the mean trait values and trait variances of the trait distributions of prey edibility, $\phi$, and of predator selectivity, $\omega$, as a function of time. Note the large difference, i.e., of two orders of magnitude, in the time scales of the two types of models. Bottom: The panels show a sequence of four snapshots of the trait distribution of prey (biomass density as a function of edibility, $\phi$, in green), and predators (biomass density as a function of selectivity, $\omega$, in blue) in the FTD model. The snapshots correspond to the times labeled and marked by vertical dotted lines in the top left panel

Fig. 6 Long-term solutions show complex predator-prey dynamics in the FTD model but regular cycles in the aggregate model under disruptive selection $(\beta=5)$, for solutions in the basin of attraction of "state I" (see Fig. 3). For detailed explanations of the panels and coloring, see legend of Fig. 4
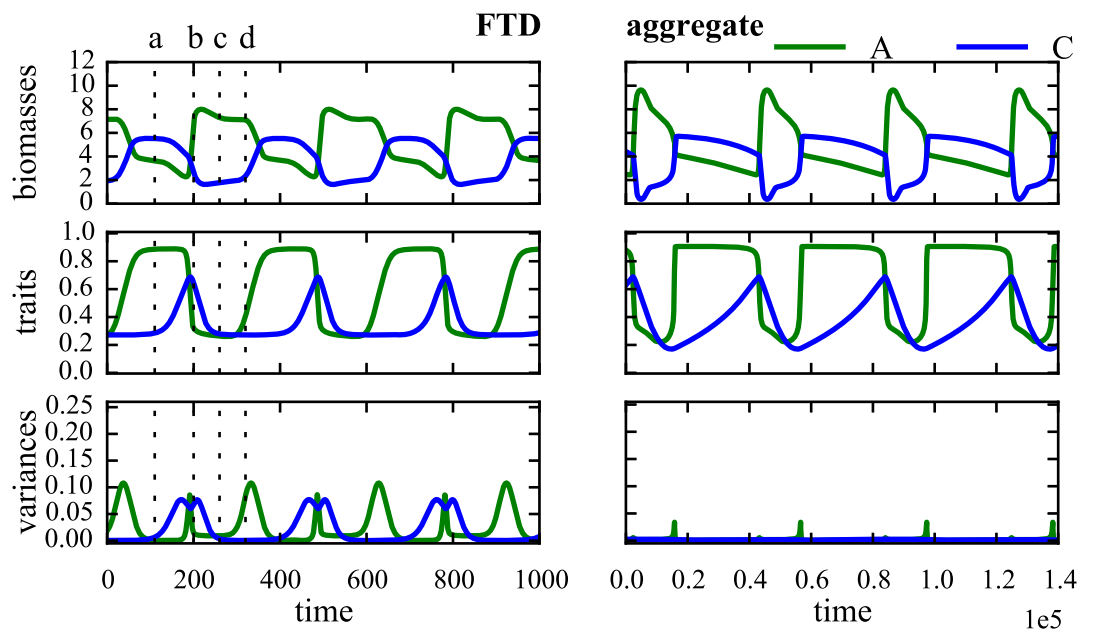

Trait distributions
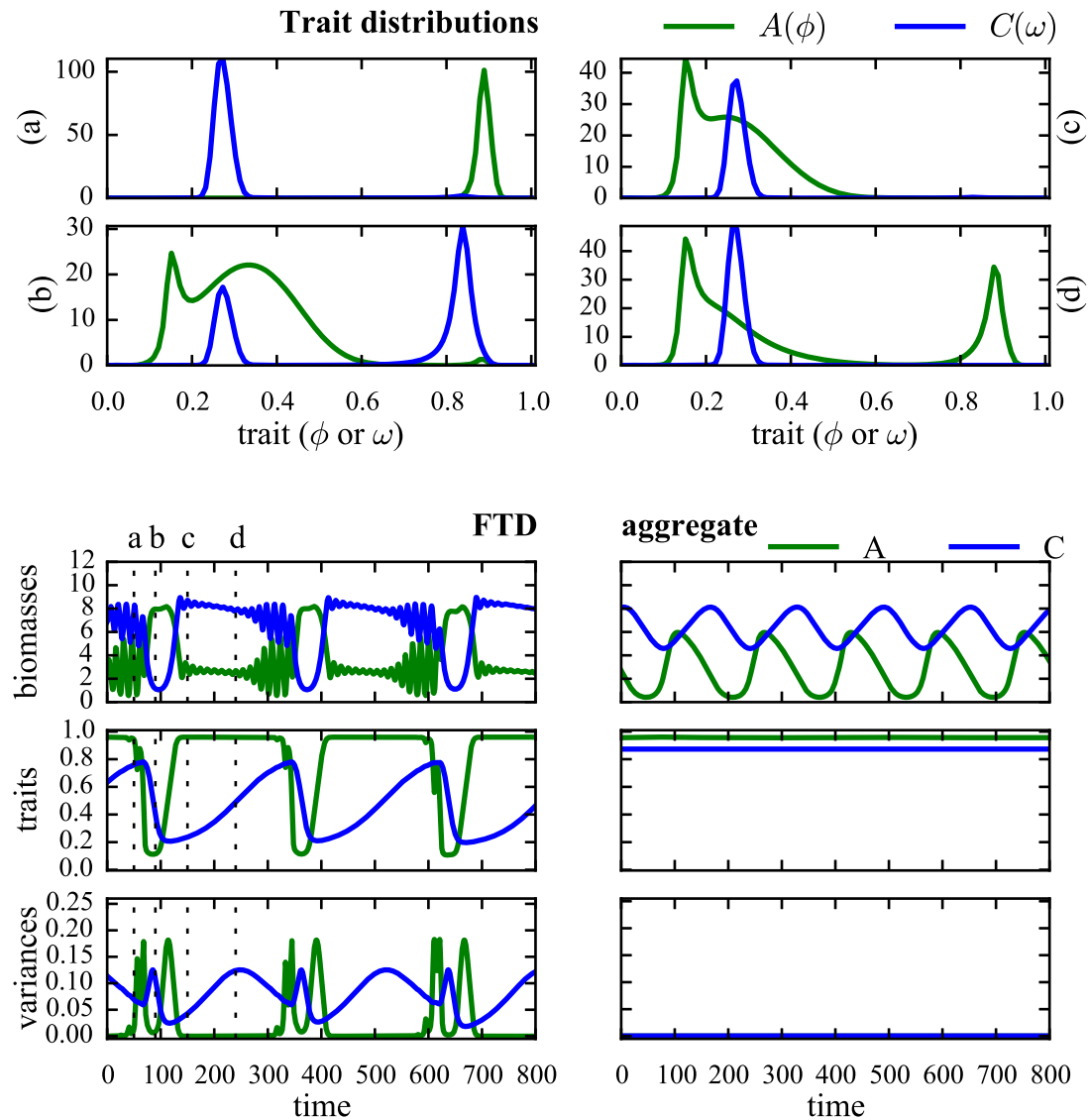

Trait distributions
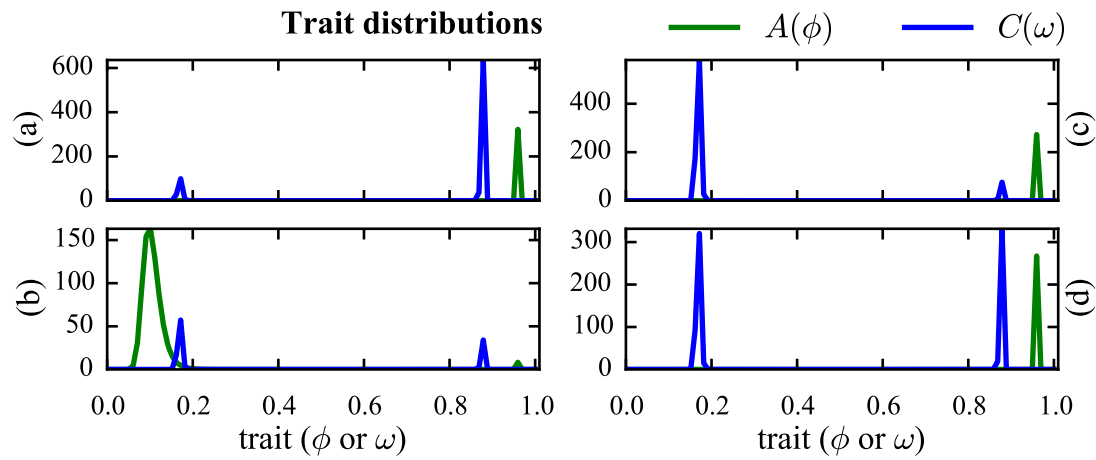
Fig. 7 Long-term solutions show stasis in both prey and predator communities under disruptive selection $(\beta=3)$ for both models in the "state II" (Fig. 3) basin of attraction. For detailed explanations of the panels and coloring, see legend of Fig. 4
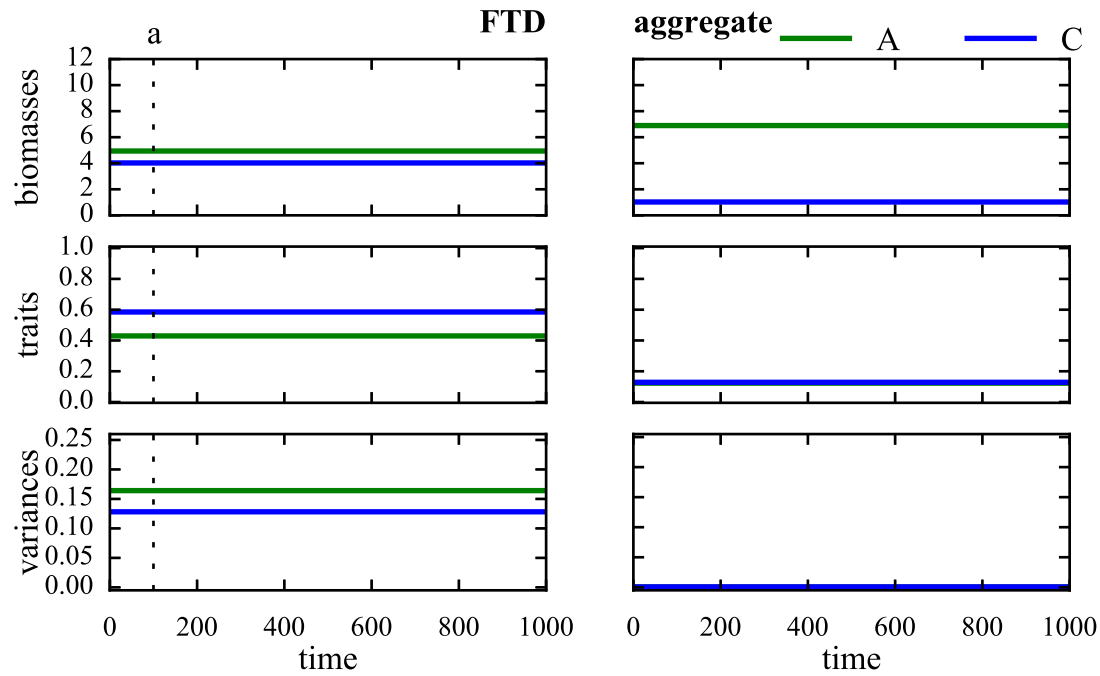

Trait distribution

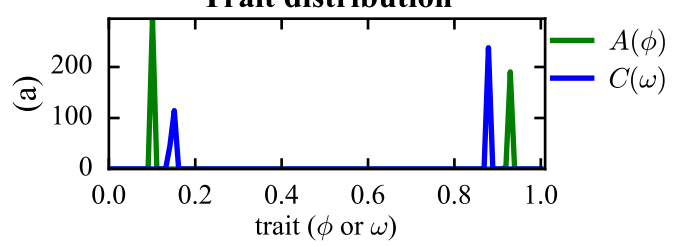

remains in the aggregate model. In addition, when the alternative states are evaluated separately, the aggregate model predicts less temporal variation in the aggregate properties than the FTD model, and thus overestimates the stability of the trait and biomass dynamics.

\section{Discussion}

Community ecology is recognizing that standing trait variation has to be incorporated into models in order to predict the behavior of food web dynamics (Norberg et al. 2001; Tirok et al. 2011). However, ecology inherited from population genetics the early view that stabilizing or weak directional selection prevails in natural communities promoting a distribution of phenotypic traits that follows closely the optimum of the fitness landscape (Lande 1982; Abrams et al. 1993). This prevalent view has facilitated the frequently made assumption of normally distributed trait values with small variances in aggregate models (Wirtz and Eckhardt 1996; Merico et al. 2009). However, there is ample empirical evidence for strong directional and disruptive selection promoting trait distributions much different from a normal distribution, questioning the strong assumptions made in aggregate models. For instance, directional selection is evidenced by the well-established alterations in phytoplankton and zooplankton composition (Sommer et al. 2012; Tirok and Gaedke 2007), where recurrent changes in the abiotic and biotic environment favor different species and trait values at different times (Vasseur and Gaedke 2007; Fox et al. 2010). Disruptive selection occurs at the population level (Kingsolver et al. 2001; Martin and Pfennig 2012), and also at the scale of whole communities where greatly different strategies coexist, e.g., small (edible) and large (less edible) phytoplankton species during summer (Sommer et al. 2012). As a consequence, a wide range of size distributions was observed within aquatic (Chisholm 1992; Tackx et al. 1994; Havlicek and Carpenter 2001; Schartau et al. 2010) and terrestrial (Maurer et al. 2004; Thibault et al. 2011) communities that exhibited large skewness and multiple modes. In addition, trait distributions may show very large variances as exemplified by phytoplankton and zooplankton size distributions (Gaedke 1992; Vergnon et al. 2009; Segura et al. 2013; Downing et al. 2014).

Hence, we investigated the reliability of the aggregate model approach for different selection regimes. As expected, stabilizing selection resulted in narrow, singlepeaked trait distributions and, thus, the aggregate model captured the features of the full trait distribution (FTD) model very well, which is in line with Merico et al. (2009). Under directional selection, our two models delivered similar kinds of oscillations and averages of the biomass and mean trait values in accordance with Norberg et al. (2001) and Terseleer et al. (2014). However, the FTD model predicted much higher trait variances than the aggregate model, resulting in major discrepancies in the timescales of their oscillations. This happens because the trait distribution in the FTD model tunnels from one end of the interval to the 
other without ever having its mode in the middle, because as soon as selection favors a trait far from that currently abundant, a population with the now-favored trait expands, promoting temporarily broad, bimodal trait distributions (for an animation of the temporal dynamics see Online Resource 1). The persistently high trait variances in the FTD model suggest that mutual adjustment between prey and predators is able to maintain functional trait diversity at both trophic levels by endogenous forces. In contrast, in the aggregate model, the mean trait has to follow one mode, i.e., a local maximum of the fitness landscape, so that it goes through all intermediate values separating the fitness peaks whenever the direction of selection changes. Unfortunately, close to a maximum, the second derivative is negative, leading to a decline of the trait variance in the aggregate model approach. Since the variance scales the rate of change of the mean trait value, trait adaptation slows down. In order to maintain high trait variances and sufficiently fast trait adaptation in the aggregate model, the mean trait value needs to stay close to a fitness minimum, where the second derivative is positive, something the local fitness maximization approach selects against. Hence, functional diversity could only be maintained through a strict balance over time between well- and maladapted mean trait values, which may rarely occur in both ecological models and natural systems. The strong constraints of aggregate models thus promote the loss of trait variance when no other source of variation is accounted for (Merico et al. 2014). To compensate for this shortcoming, previous studies introduced other ecological processes, such as additional trade-offs (Tirok et al. 2011) or trait diffusion mimicking mutation (Merico et al. 2014) which increases model complexity and may obscure real processes responsible for the maintenance of functional diversity.

The systematic underestimation of the trait variance by aggregate models may cause serious problems when aiming for the quantitative representation of food web dynamics in real ecosystems. For example, combining the aggregate model approach with observed seasonal forcing likely results in a mismatch between trait and biomass dynamics, since the small standing variance does not allow a sufficiently fast adaptation. As a result, the temporal variation in mean trait values and trait variances is underestimated. This reasoning is in line with Savage et al. (2007), who showed that increasing the amplitude or frequency of environmental fluctuations, and thus the strength of directional selection, amplified the oscillations of the mean and variance and reduced the match between their multispecies model and its moment closure approximation. Despite this, aggregate models were successfully incorporated into larger models used to address more general questions in ecosystem dynamics (Wirtz and Eckhardt 1996; Terseleer et al. 2014; Acevedo-Trejos et al. 2015). However, the timescales of their trait dynamics had to be corrected by fitting the immigration rates needed to obtain the required level of standing trait variance or by keeping the variance constant. Although the model of Terseleer et al. (2014) tracked changes in the biomass and mean cell size of a diatom community quite well, it strongly underestimated the temporal variation of the standing trait variance. Indeed, under (recurrent) directional selection, fitting higher order central moments such as skewness and kurtosis was necessary for a good approximation of the temporal dynamics of the trait variance (Norberg et al. 2001). However, a high immigration rate was still needed to get a high average value of the trait variance, and therefore the right timescale.

The predictions of our two models differed most strongly for disruptive selection, both in terms of the overall magnitude of the aggregate properties and their temporal dynamics. Disruptive selection consistently favored extreme trait values compared to intermediate ones giving rise to persistent bimodal trait distributions with large variances in the FTD model. In the aggregate model, a co-occurrence of biomass peaks at different trait values is impossible, making this approach especially conducive to large errors in such cases when higher-order moments are neglected. Accordingly, the aggregate model either strongly over- or underestimated the prey or predator biomasses. This may have a strong impact on the food web structure, the energy transfer from one trophic level to the next, and related ecosystem services, when implementing the aggregate model approach into global ocean or other ecosystem models tailored to specific systems. Trait variation was also incorporated into fishery management models using an aggregate approach (Akpalu 2009). However, our results lead us to the expectation that, depending on the underlying trade-offs of the system, a FTD model may deliver strongly deviating predictions concerning the sustainable yield of fish, with major economic and ecological consequences.

Hence, it is important to establish robust model approaches incorporating trait variation based on observable data. This demands to understand precisely the limitations of characterizing the functional trait diversity of whole communities only in terms of aggregate measures. Accordingly, recent model studies applied the aggregate model framework to functional groups instead of entire communities (Norberg et al. 2001; Terseleer et al. 2014), or combined the approach of quantitative genetics with a multispecies model approach (Norberg et al. 2012). However, it can be challenging in practice to define functional groups that are not too numerous but sufficiently cohesive so that their fitness landscapes are not subject to disruptive selection pressure. Even at the population level, frequency-dependent selection can split a sexual population into separate phenotypic clusters when mating is assortative (Doebeli 1996; Doebeli et al. 2007). Another option might be to dynamically 
model higher-order moments, such as the third and fourth ones. However, model complexity increases very quickly, the quality of the aggregate model approximation strongly decreases with increasing moment order (Bruggeman and Kooijman 2007; Savage et al. 2007), and even the direct fitting of those higher-order moments was not able to keep trait variances high (Norberg et al. 2001).

Our FTD model offers a promising alternative tool to account for the inherent flexibility in natural populations and communities. In line with Abrams (2006) and Maharjan et al. (2013), we show that the shape of trade-offs strongly influences the manifold interactions between all the different phenotypes in our predator-prey system allowing for stabilizing, directional or even disruptive selection. In the last two cases, this frequently leads to bimodal trait distributions with large variance implying that the maintenance of diversity is related to the complex interactions between the ubiquitous non-linearities in the growth and death rates of populations and the diversity of their traits (see Eq. 24). This fits with recent studies linking frequency-dependent interactions to pattern formation in phenotypic space in the form of multimodal trait distributions (Dieckmann et al. 2004; Allen et al. 2006; Doebeli et al. 2007; Pigolotti et al. 2010). However, in contrast to previous studies, we consider in addition to direct resource competition (Scheffer and van Nes 2006; Doebeli et al. 2007) also apparent competition. Hence, trophic interactions such as prey and predator relationships may also give rise to lumpiness in pattern formation as already pointed out in the prospective study of Holling (1992). This agrees with findings from an NPZ (nutrient-phytoplankton-zooplankton) model, showing persistent multimodal size distributions within phytoplankton and zooplankton communities when accounting for empirical allometric relationships in their growth and loss rates (Banas 2011). In our FTD model, the emergent trait distributions in the prey and predator communities are very similar because both trophic levels are strongly linked. This may explain the very similar size distributions in herbivores and carnivores in natural systems (Holling 1992). Hence, our FTD model predicts a testable relationship between trade-off shapes and forms of functional trait distributions, which may encourage future empirical studies to measure the shape of trade-offs and relate it to observed trait distributions. Although the shapes of the trade-offs are pivotal in determining the dynamics and diversity of the modeled ecosystems, empirical data on such relationships is scant (but see Maharjan et al. 2013).

The formation of bimodal trait distributions in our FTD model under directional and disruptive selection was stabilized but not caused by immigration ("Appendix B: Model version with mutation"; for an animation of the temporal dynamics, see also Online Resource 2). Indeed, a FTD model version without immigration but with mutation, modeled as trait diffusion, maintained the main features of our original FTD model. In contrast, the corresponding aggregate model deviated both from its FTD analogue as well as from the model version with immigration ("Appendix B: Model version with mutation"). This shows that aggregate approaches have also to be careful about the choice of mechanism responsible for maintaining diversity (variance), as the predictions are not robust to those choices.

In conclusion, community and ecosystem models need to account for the possibility of strongly non-linear fitness landscapes that may give rise to bimodal trait distributions with large variances, rendering aggregate models inaccurate. The common practice to consider only the fitness landscape close to the optimum trait has to be questioned and carefully assessed in each case. Aggregate models approximate trait dynamics well under specific conditions, such as stabilizing selection, but fail to do so if largely different trait values prevail simultaneously within the community or functional group considered. Hence, with the FTD model presented, we are better equipped to reveal the mechanisms underlying the maintenance of functional diversity in traits.

Acknowledgments We thank E. van Velzen and two anonymous referees for helpful comments and suggestions. RMC was supported through grant \#2012/05949-6, São Paulo Research Foundation (FAPESP), Brazil, and also through a post-doctoral PNPD fellowship from CAPES (Brazil). TK was funded by the German Research Foundation (DFG, GA 401/19-1 and 26-1).

\section{Appendix A: Derivation of the aggregate model}

The FTD model describes the temporal changes in the biomass density of each particular trait value and thus yields, at every moment in time, the biomass densities of the prey and predator communities (or functional groups) as a function of their respective traits $\phi$ and $\omega$. As a consequence, the magnitude and shape of the biomass-trait distributions may change in time. Instead of resolving the rate of change of the biomass density for each particular trait value, one may directly describe the temporal changes in the aggregate properties of the prey and predator communities using moment approximation methods. In this case, the dynamics of the FTD model is approximated by a corresponding aggregate model which tracks only the temporal changes in the total biomasses of the prey and predator communities and the locations (mean trait values) and widths (trait variances) of the corresponding trait distributions. These aggregate quantities are related to the trait distribution by integrals over the ecologically feasible trait range, in accordance with Eq. 5. In this way, aggregate models explicitly represent the trait-dependent growth and loss terms that determine the dynamics of the aggregate properties of the trait distribution (cf. Tirok et al. 2011). 
To derive the aggregate model (6-8) from the FTD model (1-3), one has to write down the rate of change of the aggregate properties using Eqs. 1-3 and expand all functions in Taylor series around the mean trait value, since the average over the distribution of the per-capita net growth rates determines the overall trait and biomass dynamics. This leads to expressions written in terms of (central) moments of the trait distributions. Afterwards, a moment approximation is performed by truncating these series up to the second order. This will be a good approximation when the growth and grazing functions have only weak non-linearities and thus all higher-order derivatives quickly vanish, or when the trait distribution is very narrow, that is, has a small variance. Hence, the error in the biomass and trait dynamics will be most pronounced if both the higher-order derivatives of the fitness landscape, i.e., the per-capita net-growth rate as a function of the trait, evaluated at the mean trait value and the higher-order moments of the trait distribution obtain large absolute values at the same time. For example, if trait distributions with a sufficiently large variance exhibit large values of skewness or kurtosis, the quality of the approximation made by the corresponding aggregate model will be rather poor when the fitness landscape is highly non-linear. This holds since the performance of species with trait values away from the community average $\bar{\phi}$ will likely differ from the performance of a species with a trait value equal to $\bar{\phi}$ (cf. Ruel and Ayres 1999; Tirok et al. 2011).

For sake of brevity, we will derive the prey equations in detail below, as the derivation of the equations for the predator are completely analogous. The Taylor expansion of a function $f$ around the prey's mean trait value $\bar{\phi}$ is given by:

$f(\phi)=\sum_{n=0}^{\infty} \frac{(\phi-\bar{\phi})^{n}}{n !} \frac{d^{n} f(\bar{\phi})}{d \phi^{n}}$.

Since we are dealing with aggregate quantities such as prey biomass summed over the whole trait range, we will need the expansion of integrals over the prey's trait distribution $A(\phi)$, such as:

$$
\begin{aligned}
\int f(\phi) A(\phi) d \phi & =\int \sum_{n=0}^{\infty} \frac{(\phi-\bar{\phi})^{n}}{n !} A(\phi) \frac{d^{n} f(\bar{\phi})}{d \phi^{n}} d \phi \\
& =A_{T} \sum_{n=0}^{\infty} \frac{1}{n !} \frac{d^{n} f(\bar{\phi})}{d \phi^{n}} M_{n} \\
& =A_{T}\left[f(\bar{\phi})+f^{\prime}(\bar{\phi}) M_{1}^{\sigma^{0}}+\frac{f^{\prime \prime}(\bar{\phi})}{2} M_{2}+\cdots\right],
\end{aligned}
$$

where $M_{n}$ is the $n$th central moment of the distribution $A(\phi)$ (in particular, $M_{2}=v_{\phi}$ ), defined by

$$
M_{n}=\frac{1}{A_{T}} \int(\phi-\bar{\phi})^{n} A(\phi) d \phi .
$$

The rate of change of the total prey biomass $A_{T}$ is

$$
\begin{aligned}
\frac{d A_{T}}{d t} & =\frac{d}{d t} \int A(\phi) d \phi=\int \frac{d A(\phi)}{d t} d \phi \\
& =\int\left[A(\phi) \tilde{R}(\phi)+I_{A}\right] d \phi
\end{aligned}
$$

where $\tilde{R}$ is the per capita net growth rate from Eq. 1:

$$
\tilde{R}(\phi)=r(\phi)+B(\phi)-\frac{1}{A(\phi)} \int g(\phi, \omega) C(\omega) d \omega
$$

Now, the grazing term already contains an integral over $\phi^{\prime}$, independent of $\phi$, so we expand it first:

$$
\begin{aligned}
& \frac{1}{A(\phi)} \int g(\phi, \omega) C(\omega) d \omega \\
= & \frac{g_{m} q(\phi, \omega)}{M(\omega)+\int q\left(\phi^{\prime}, \omega\right) A\left(\phi^{\prime}\right) d \phi^{\prime}} \\
= & \frac{g_{m} q(\phi, \omega)}{M(\omega)+A_{T}\left[q(\bar{\phi}, \omega)+\left.\frac{\nu_{\phi}}{2} \frac{\partial^{2} q(\phi, \omega)}{\partial \phi^{2}}\right|_{\phi=\bar{\phi}}+\ldots\right]},
\end{aligned}
$$

and here we drop terms of order higher than two (denoted by the dots), which gives us simply $G(\phi, \omega)$ defined by Eq. 8 . We now proceed expanding the integral in terms of $\omega$ :

$$
\begin{aligned}
\tilde{R}(\phi)= & r(\phi)+B(\phi)-\int G(\phi, \omega) C(\omega) d \omega \\
= & r(\phi)+B(\phi) \\
& +C_{T}\left[G(\phi, \bar{\omega})+\left.\frac{v_{\omega}}{2} \frac{\partial^{2} G(\phi, \omega)}{\partial \omega^{2}}\right|_{\omega=\bar{\omega}}+\ldots\right],
\end{aligned}
$$

and, stopping once again at the second order, we arrive at the expression for $R_{A}(\phi)$ (7). Substituting it back into Eq. 15 and expanding:

$$
\begin{aligned}
\frac{d A_{T}}{d t} & =\int\left[A(\phi) R_{A}(\phi)+I_{A}\right] d \phi \\
& =A_{T}\left(R_{A}(\bar{\phi})+\left.\frac{v_{\phi}}{2} \frac{\partial^{2} R_{A}}{\partial \phi^{2}}\right|_{\phi=\bar{\phi}}+\cdots\right)+I_{A},
\end{aligned}
$$

which yields Eq. 6 when we truncate at the second order. 
The rate of change of the mean trait value is derived in a similar fashion:

$$
\begin{aligned}
\frac{d \bar{\phi}}{d t}= & \frac{1}{A_{T}}\left[\frac{d}{d t} \int \phi A(\phi) d \phi-\frac{1}{A_{T}} \frac{d A_{T}}{d t} \int \phi A(\phi) d \phi\right] \\
= & \frac{1}{A_{T}}\left[\int \phi \frac{d A(\phi)}{d t} d \phi-\bar{\phi} \frac{d A_{T}}{d t}\right] \\
= & \frac{1}{A_{T}} \int(\phi-\bar{\phi}) \frac{d A(\phi)}{d t} d \phi \\
= & \frac{1}{A_{T}} \int(\phi-\bar{\phi})\left(A(\phi) R_{A}(\phi)+I_{A}\right) d \phi \\
= & \frac{1}{A_{T}} \int(\phi-\bar{\phi}) A(\phi) R_{A}(\phi) d \phi \\
& +\frac{1}{A_{T}} \int(\phi-\bar{\phi}) I_{A} d \phi \\
= & \left(\left.v_{\phi} \frac{\partial R_{A}}{\partial \phi}\right|_{\phi=\bar{\phi}}+\left.\frac{M_{3}}{2} \frac{\partial^{2} R_{A}}{\partial \phi^{2}}\right|_{\phi=\bar{\phi}}+\ldots\right) \\
& +\frac{I_{A}}{A_{T}}\left(\frac{1}{2}-\bar{\phi}\right) \cdot
\end{aligned}
$$

Finally, the trait variance will be expressed by:

$$
\begin{aligned}
\frac{d v_{\phi}}{d t}=\frac{1}{A_{T}} & {\left[\frac{d}{d t} \int(\phi-\bar{\phi})^{2} A(\phi) d \phi\right.} \\
& \left.-\frac{1}{A_{T}} \frac{d A_{T}}{d t} \int(\phi-\bar{\phi})^{2} A(\phi) d \phi\right] \\
=\frac{1}{A_{T}} & {\left[\int(\phi-\bar{\phi})^{2} \frac{d A(\phi)}{d t} d \phi-v_{\phi} \frac{d A_{T}}{d t}\right] . }
\end{aligned}
$$

The second term can be expanded directly using Eq. 19

$$
\frac{v_{\phi}}{A_{T}} \frac{d A_{T}}{d t}=v_{\phi}\left[\left(R_{A}(\bar{\phi})+\left.\frac{v_{\phi}}{2} \frac{\partial^{2} R_{A}}{\partial \phi^{2}}\right|_{\phi=\bar{\phi}}+\cdots\right)+\frac{I_{A}}{A_{T}}\right],
$$

while the first term can be written as:

$$
\begin{aligned}
& \frac{1}{A_{T}} \int(\phi-\bar{\phi})^{2}\left(A(\phi) R_{A}(\phi)+I_{A}\right) d \phi=v_{\phi} R_{A}(\bar{\phi}) \\
& +\left.M_{3} \frac{\partial R_{A}}{\partial \phi}\right|_{\phi=\bar{\phi}}+\left.\frac{M_{4}}{2} \frac{\partial^{2} R_{A}}{\partial \phi^{2}}\right|_{\phi=\bar{\phi}} \\
& +\frac{I_{A}}{A_{T}}\left(\frac{1}{3}+\bar{\phi}^{2}-\bar{\phi}\right) .
\end{aligned}
$$

Given the fact that the terms proportional to $v_{\phi}$ in Eq. 23 cancel with the ones from Eq. 22 and that the terms proportional to $v_{\phi}^{2}$ combine with those from Eq. 22, the rate of change of the trait variance can be approximated by:

$$
\begin{aligned}
\frac{d \nu_{\phi}}{d t}= & \left.M_{3} \frac{\partial R_{A}}{\partial \phi}\right|_{\phi=\bar{\phi}}+\left.\frac{\left(M_{4}-v_{\phi}^{2}\right)}{2} \frac{\partial^{2} R_{A}}{\partial \phi^{2}}\right|_{\phi=\bar{\phi}} \\
& +\frac{I_{A}}{A_{T}}\left[\frac{1}{12}-v_{\phi}+\left(\frac{1}{2}-\bar{\phi}\right)^{2}\right] .
\end{aligned}
$$

As seen in Eqs. 20 and 24, temporal changes in the aggregate properties (lower-order moments) generally depend on higher-order moments. To derive self-contained expressions for the evolution of the aggregate properties, and thus to close the system of differential equations, one has to describe the higher-order moments in the corresponding equations by lower-order moments. Different moment closure techniques were described in detail by Norberg et al. (2001) and Merico et al. (2009). For instance, Norberg et al. (2001) described the third and fourth central moments by power-functions of the first (mean trait) and second (variance) central moments and the optimal trait value. The parameters were estimated from the trait distributions obtained from simulations of the underlying multispecies model. Another approach is to assume the trait distributions to be well represented by a Gaussian (Wirtz and Eckhardt 1996; Merico et al. 2009; Tirok et al. 2011) for which the higher-order moments are either zero (odd moments) or fully determined by the variance alone (even moments).

Following Wirtz and Eckhardt (1996) and Merico et al. (2009), we assume traits to be normally distributed. Using the fact that the third central moment of a Gaussian distribution equals zero $\left(M_{3}=0\right.$; the normal distribution is symmetric), and that its fourth central moment is proportional to the square of its variance $\left(M_{4}=3 M_{2}^{2}\right)$, that is, its excess kurtosis is 0 , we can approximate the rate of change of the mean trait value Eq. 20 and of the trait variance Eq. 24 by Eq. 6.

\section{Appendix B: Model version with mutation}

The immigration term in Eqs. 1 and 6 prevents the extinction of species by providing a very small constant influx of immigrants of all trait values. This is generally realistic and necessary to ensure that some variance is retained in the aggregate model, but may in principle strongly influence the dynamics of both types of models. Here, we build versions of the FTD model and the aggregate model without immigration, but with mutation instead. Mutation can be an important process when dealing with narrowly defined functional groups, where the trait variance may originate mostly from intra-populational processes rather than standing trait variance among different species.

Here, mutation is understood as a small probability of offspring to have trait values slightly different then their parents (we do not account for sexual mixing). It is modeled as a (Fokker-Planck) diffusive term, corresponding to the second derivative of the population density times the birth rate with respect to the trait value. In the case of prey, we assign all density-dependent terms to the mortality, and thus their birth rate, $\rho_{A}$, is simply the intrinsic growth rate $\left(r^{\prime}\right)$. The 
birth rate $\rho_{C}$ of predators is their grazing rate. This leads to the modified FTD model:

$$
\begin{aligned}
\frac{\partial A(\phi, t)}{\partial t}= & (r(\phi)+B(\phi)) A-\int g(\phi, \omega) C d \omega \\
& +\mu_{A} \frac{\partial^{2}\left[\rho_{A} A(\phi)\right]}{\partial \phi^{2}} \\
\frac{\partial C(\omega, t)}{\partial t}= & \left(e \int g(\phi, \omega) d \phi-d+B(\omega)\right) C \\
& +\mu_{C} \frac{\partial^{2}\left[\rho_{C} C(\omega)\right]}{\partial \omega^{2}} \\
\rho_{A}(\phi)= & r^{\prime}(\phi) \quad \rho_{C}(\omega)=e \int g(\phi, \omega) d \phi,
\end{aligned}
$$

where $\mu_{A}$ and $\mu_{C}$ are the mutation rates for prey and predator, respectively, and all other terms and parameters are as before. We derive the aggregate model from the FTD model from this equation together with Eq. 5. Due to the boundary functions imposed, the population densities go to zero very quickly outside the range of traits from 0 to 1 . Therefore, we assume that $A(\phi)$, as well as its derivatives, go fast enough to 0 as $\phi$ goes to (plus or minus) infinity, which guarantees that terms calculated at infinity appearing via integration by parts vanish.

The derivation proceeds exactly as in "Appendix A: Derivation of the aggregate model". We begin by calculating the rate of change of total biomass, $\frac{d A_{T}}{d t}$. The new term corresponding to mutation in Eq. 15 will be:

$\mu_{A} \int \frac{\partial^{2}\left[\rho_{A} A(\phi)\right]}{\partial \phi^{2}} d \phi=\left.\mu_{A} \frac{\partial\left[\rho_{A} A(\phi)\right]}{\partial \phi}\right|_{-\infty} ^{\infty}=0$

which means that mutation does not contribute any extra term to the total biomass equation. Proceeding to the mean trait equation, $\frac{d \bar{\phi}}{d t}$, we now get the following contribution of the mutation term:

$$
\begin{aligned}
\frac{\mu_{A}}{A_{T}} \int \phi \frac{\partial^{2}\left[\rho_{A} A(\phi)\right]}{\partial \phi^{2}} d \phi= & \left.\frac{\mu_{A}}{A_{T}}\left[\phi \frac{\partial\left[\rho_{A} A(\phi)\right]}{\partial \phi}\right]\right|_{-\infty} ^{\infty} \\
& -\frac{\mu_{A}}{A_{T}} \int \frac{\partial\left[\rho_{A} A(\phi)\right]}{\partial \phi} d \phi \\
= & 0-\left.\frac{\mu_{A}}{A_{T}}\left[\rho_{A} A(\phi)\right]\right|_{-\infty} ^{\infty} \\
= & 0,
\end{aligned}
$$

and hence mutation does not affect the mean trait value either. Finally, the equation for the rate of change of the trait variance, $\frac{d v_{\phi}}{d t}$, has a term due to mutation given by:

$$
\begin{aligned}
& \frac{\mu_{A}}{A_{T}} \int \phi^{2} \frac{\partial^{2}\left[\rho_{A} A(\phi)\right]}{\partial \phi^{2}} d \phi=\left.\frac{\mu_{A}}{A_{T}}\left[\phi^{2} \frac{\partial\left[\rho_{A} A(\phi)\right]}{\partial \phi}\right]\right|_{-\infty} ^{\infty} \\
& -2 \frac{\mu_{A}}{A_{T}} \int \phi \frac{\partial\left[\rho_{A} A(\phi)\right]}{\partial \phi} d \phi \\
= & 0-2 \frac{\mu_{A}}{A_{T}}\left\{\left.\left[\phi \rho_{A} A(\phi)\right]\right|_{-\infty} ^{\infty}-\frac{\mu_{A}}{A_{T}} \int \rho_{A} A(\phi) d \phi\right\} \\
= & 2 \frac{\mu_{A}}{A_{T}} \int \rho_{A} A(\phi) d \phi \\
= & 2 \mu_{A}\left[\rho_{A}(\bar{\phi})+\left.\frac{v_{\phi}}{2} \frac{\partial^{2} \rho_{A}(\phi)}{\partial \phi^{2}}\right|_{\phi=\bar{\phi}}+\ldots\right] .
\end{aligned}
$$

Thus the equation for trait variance becomes:

$$
\frac{d v_{\phi}}{d t}=\left.v_{\phi}^{2} \frac{\partial^{2} R_{A}}{\partial \phi^{2}}\right|_{\phi=\bar{\phi}}+2 \mu_{A} \rho_{A}(\bar{\phi})+\left.v_{\phi} \mu_{A} \frac{\partial^{2} \rho_{A}(\phi)}{\partial \phi^{2}}\right|_{\phi=\bar{\phi}} .
$$

We analyzed the resulting dynamics of the FTD model and the aggregate model as described in the methods of the main text. For all selection regimes, Fig. 8 shows very similar patterns for the FTD model with mutation compared to the FTD model with immigration (cf. Fig. 3). In contrast, the aggregate model no longer displayed large oscillations in its mean trait values for intermediate values of $\beta$, but high trait variances instead, indicating that even the type of dynamics it generates is not robust to such structural modification. Importantly, immigration influenced the mean trait values directly, pulling toward intermediate values and facilitating recurrent changes in trait values. Even for stabilizing selection, the agreement between the two types of models in the temporal averages of the total biomasses and mean trait values is worse than in the scenario with immigration, since variance levels of the FTD model are higher in the mutation variant $\left(4 \times 10^{-3}\right.$ compared to $2 \times 10^{-5}$, when $\left.\beta=0.2\right)$, leading to worse performance of the aggregate model due to Jensen's inequality.

In the absence of immigration, the FTD model no longer exhibited bistability. Also, the transition between states of very different dominant trait values occurred more slowly, leading to longer cycles. Nonetheless, it still presented bimodal trait distributions for a wide range of parameters. For instance, $P$ was above 1.2 (see "Numerical simulations and data analysis" section) during 12 and $68 \%$ of the time for prey and predators, respectively, with $\beta=3$, and during 5 and $45 \%$ for $\beta=5$. This confirms that bimodal trait distributions were stabilized but not caused by immigration, which is in accordance with findings from Pigolotti et al. (2010). 
Fig. 8 Temporal averages (lines) and variation $( \pm 1$ standard deviation; shades) of biomass, mean trait, and standing trait variance of the solutions of the FTD (red) and aggregate (green) models with mutation instead of immigration $(25,29)$, as the shape of the trade-offs $(\beta)$ is varied. Mutation rates were set to $\mu_{A}=10^{-4}$ and $\mu_{C}=2 \times 10^{-5}$, with all other parameters as in Table 1. The dotted lines correspond to alternative long-term solutions, obtained by exploring different initial conditions, leading to distinct basins of attraction

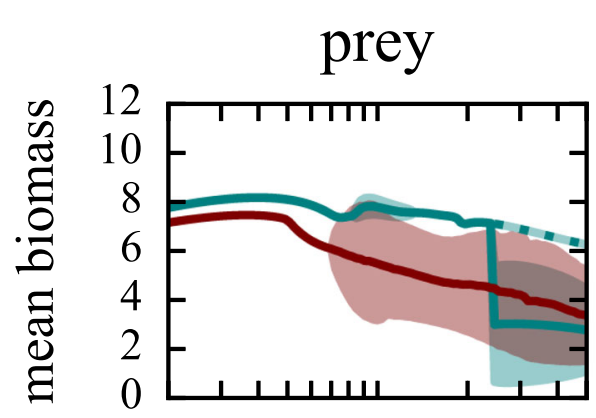

predator
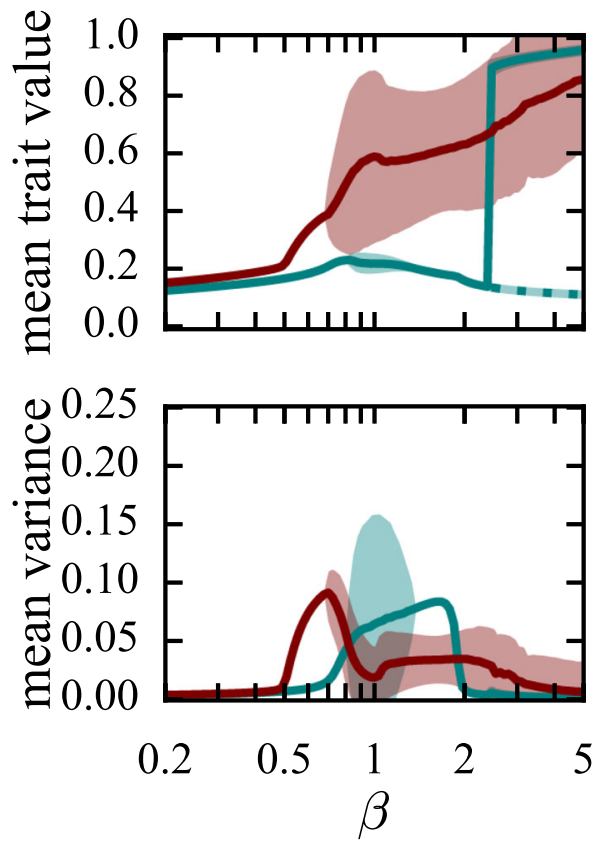
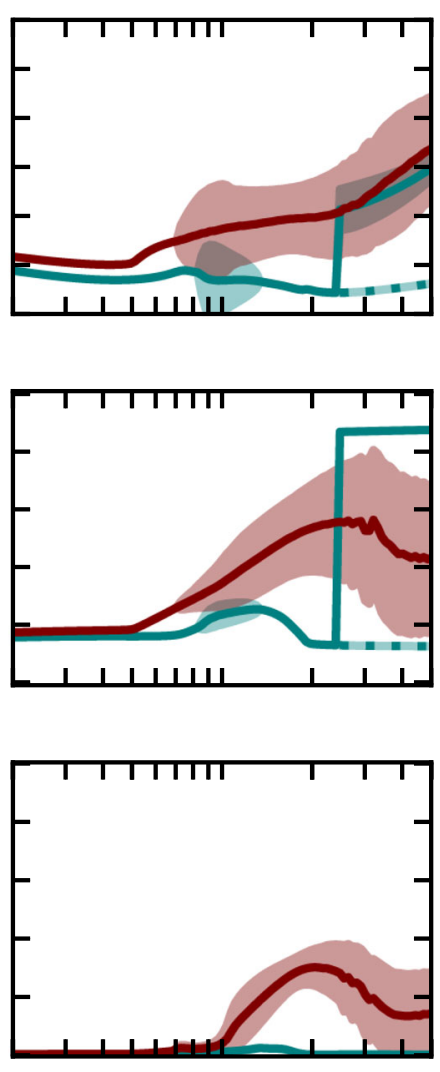

0.2

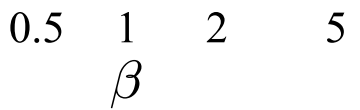

\section{References}

Abrams PA (2006) The prerequisites for and likelihood of generalistspecialist coexistence. Am Nat 167:329-342

Abrams PA, Harada Y, Matsuda H (1993) On the relationship between quantitative genetic and ESS models. Evolution 47:982-985

Acevedo-Trejos E, Brandt G, Bruggeman J, Merico A (2015) Mechanisms shaping size structure and functional diversity of phytoplankton communities in the ocean. Scientific reports 5

Akpalu W (2009) Economics of biodiversity and sustainable fisheries management. Ecol Econ 68(10):2729-2733

Allen CR, Garmestani A, Havlicek T, Marquet P, Peterson G (2006) Patterns in body mass distributions: sifting among alternative hypotheses. Ecol Lett 9:630-643

Banas NS (2011) Adding complex trophic interactions to a sizespectral plankton model: emergent diversity patterns and limits on predictability. Ecol Model 222:2663-2675

Baptestini EM, de Aguiar MA, Bolnick DI, Araujo MS (2009) The shape of the competition and carrying capacity kernels affects the likelihood of disruptive selection. J Theor Biol 259:5-11

Bauer B, Vos M, Klauschies T, Gaedke U (2014) Diversity, functional similarity, and top-down control drive synchronization and the reliability of ecosystem function. Am Nat 183(3):394-409

Becks L, Ellner SP, Jones LE, Hairston Jr. NG (2010) Reduction of adaptive genetic diversity radically alters eco-evolutionary community dynamics. Ecol Lett 13(8):989-997
Blest DC (2003) A new measure of kurtosis adjusted for skewness. Australian \& New Zealand J Stat 45(2):175-179

Bruggeman J, Kooijman SA (2007) A biodiversity-inspired approach to aquatic ecosystem modeling. Limnol Oceanogr 52(4):15331544

Chisholm SW (1992) Phytoplankton size. In: Falkowski PG, Woodhead AD (eds) Primary productivity and biogeochemical cycles in the sea. Springer, pp 213-237

DeCarlo LT (1997) On the meaning and use of kurtosis. Psychol Methods 2(3):292

Dieckmann U, Doebeli M, Metz J, Tautz D (2004) Adaptive speciation. Cambridge University Press, Cambridge

Doebeli M (1996) A quantitative genetic competition model for sympatric speciation. J Evol Biol 9:893-909

Doebeli M, Blok HJ, Leimar O, Dieckmann U (2007) Multimodal pattern formation in phenotype distributions of sexual populations. Proc R Soc Lond B Biol Sci 274(1608):347-357

Downing A, Hajdu S, Hjerne O, Otto S, Blenckner T, Larsson U, Winder M (2014) Zooming in on size distribution patterns underlying species coexistence in baltic sea phytoplankton. Ecol Lett 17:1219-1227

Fox JW, Nelson WA, McCauley E (2010) Coexistence mechanisms and the paradox of the plankton: quantifying selection from noisy data. Ecology 91:1774-1786

Gaedke U (1992) The size distribution of plankton biomass in a large lake and its seasonal variability. Limnol Oceanogr 37(6):12021220 
Gaedke U, Wickham S (2004) Ciliate dynamics in response to changing biotic and abiotic conditions in a large, deep lake (lake constance). Aquat Microb Ecol 34:247-261

Hansen PJ, Bjornsen PK, Hansen BW (1997) Zooplankton grazing and growth: scaling within the 2-2000- $\mu \mathrm{m}$ body size range. Limnol Oceanogr 42:687-704

Havlicek TD, Carpenter SR (2001) Pelagic species size distributions in lakes: are they discontinuous? Limnol Oceanogr 46(5):1021-1033

Hindmarsh AC (1983) Odepack, a systematized collection of ode solvers. In: et al RSS (ed) Scientific computing, IMACS transactions on scientific computation, vol 1. North-Holland, Amsterdam, pp 55-64

Holling C (1965) The functional response of predators to prey density and its role in mimicry and population regulation. Memoirs of the Entomological Society of Canada 45:1-60

Holling C (1992) Cross-scale morphology, geometry and dynamics of ecosystems. Ecol Monogr 62(4):447-502

Horn H, Paul L, Horn W, Petzoldt T (2011) Long-term trends in the diatom composition of the spring bloom of a German reservoir: is aulacoseira subarctica favoured by warm winters? Freshw Biol 56:2483-2499

Jones E, Oliphant T, Peterson P, et al. (2001) SciPy: open source scientific tools for Python. http://www.scipy.org/, [Online; accessed 2015-07-25]

Jones M, Rosco J, Pewsey A (2011) Skewness-invariant measures of kurtosis. Am Stat 65(2):89-95

Kingsolver JG, Hoekstra HE, Hoekstra JM, Berrigan D, Vignieri SN, Hill C, Hoang A, Gibert P, Beerli P (2001) The strength of phenotypic selection in natural populations. Am Nat 157(3):245261

Klaassen CA, Mokveld PJ, Van Es B (2000) Squared skewness minus kurtosis bounded by $186 / 125$ for unimodal distributions. Statistics \& Probability Letters 50(2):131-135

Lande R (1982) A quantitative genetic theory of life history evolution. Ecology 63:607-615

Leibold MA, Norberg J (2004) Biodiversity in metacommunities: plankton as complex adaptive systems? Limnol Oceanogr 49:1278-1289

Levins R (1962) Theory of fitness in a heterogeneous environment. i. the fitness set and adaptive function. Am Nat 96(891):361-373

Maharjan R, Nilsson S, Sung J, Haynes K, Beardmore RE, Hurst LD, Ferenci T, Gudelj I (2013) The form of a trade-off determines the response to competition. Ecol Lett 16:1267-1276

Martin RA, Pfennig DW (2012) Widespread disruptive selection in the wild is associated with intense resource competition. BMC Evol Biol 12(1):136

Maurer BA, Brown JH, Dayan T, Enquist BJ, Ernest SM, Hadly EA, Haskell JP, Jablonski D, Jones KE, Kaufman DM, et al. (2004) Similarities in body size distributions of small-bodied flying vertebrates. Evol Ecol Res 6(6):783-797

Merico A, Bruggeman J, Wirtz K (2009) A trait-based approach for downscaling complexity in plankton ecosystem models. Ecol Model 220(21):3001-3010

Merico A, Brandt G, Smith SL, Oliver M (2014) Sustaining diversity in trait-based models of phytoplankton communities. Population Dynamics 2:59

Moors J (1986) The meaning of kurtosis: Darlington reexamined. Am Stat 40(4):283-284

Norberg J (2004) Biodiversity and ecosystem functioning: a complex adaptive systems approach. Limnol Oceanogr 49:12691277

Norberg J, Swaney DP, Dushoff J, Lin J, Casagrandi R, Levin SA (2001) Phenotypic diversity and ecosystem functioning in changing environments: a theoretical framework. Proc Natl Acad Sci 98(20):11,376-11,381
Norberg J, Urban MC, Vellend M, Klausmeier CA, Loeuille N (2012) Eco-evolutionary responses of biodiversity to climate change. Nat Clim Chang: $1-5$

Pearson K (1929) Editorial note on inequalities for moments of frequency functions and for various statistical constants. Biometrika:370-375

Pigolotti S, López C, Hernández-García E, Andersen KH (2010) How gaussian competition leads to lumpy or uniform species distributions. Theor Ecol 3:89-96

Rosenzweig M, MacArthur R (1963) Graphical representation and stability conditions of predator-prey interactions. Am Nat 97:209223

Ruel J, Ayres M (1999) Jensen's inequality predicts effects of environmental variation. TREE 14:361-366

Saloniemi I (1993) A coevolutionary predator-prey model with quantitative characters. Am Nat 141(6):880-896

Savage VM, Webb CT, Norberg J (2007) A general multi-trait-based framework for studying the effects of biodiversity on ecosystem functioning. J Theor Biol 247(2):213-229

Schartau M, Landry MR, Armstrong RA (2010) Density estimation of plankton size spectra: a reanalysis of ironex ii data. J Plankton Res 32(8):1167-1184

Scheffer M, van Nes EH (2006) Self-organized similarity, the evolutionary emergence of groups of similar species. Proc Natl Acad Sci 103(16):6230-6235

Scheffer M, Rinaldi S, Gragnani A, Mur LR, van Nes EH (1997) On the dominance of filamentous cyanobacteria in shallow, turbid lakes. Ecology 78:272-282

Segura A, Kruk C, Calliari D, García-rodriguez F, Conde D, Widdicombe C, Fort H (2013) Competition drives clumpy species coexistence in estuarine phytoplankton. Scientific reports 3

Sommer U, Adrian R, De Senerpont Domis L, Elser JJ, Gaedke U, Ibelings B, Jeppesen E, Lürling M, Molinero JC, Mooij WM, et al. (2012) Beyond the plankton ecology group (peg) model: mechanisms driving plankton succession. Annu Rev Ecol Evol Syst 43:429-448

Straub C, Ives A, Gratton C (2011) Evidence for a trade-off between host-range breadth and host-use efficiency in aphid parasitoids. Am Nat 177:389-395

Strauss S, Rudgers J, Lau J, Irwin R (2002) Direct and ecological costs of resistance to herbivory. Trends Ecol Evol 17:278-285

Tackx MLM, Herman PMJ, van Rijswijk P, Vink M, Bakker C (1994) Plankton size distributions and trophic relations before and after the construction of the storm-surge barrier in the oosterschelde estuary. Hydrobiologia 282(/283):145-152

Tang EP (1995) The allometry of algal growth rates. J Plankton Res 17:1325-1335

Terseleer N, Bruggeman J, Lancelot C, Gypens N (2014) Trait-based representation of diatom functional diversity in a plankton functional type model of the eutrophied southern north sea. Limnol Oceanogr 59(6):1958-1972

Thibault KM, White EP, Hurlbert AH, Ernest S (2011) Multimodality in the individual size distributions of bird communities. Glob Ecol Biogeogr 20(1):145-153

Tirok K, Gaedke U (2007) The effect of irradiance, vertical mixing and temperature on spring phytoplankton dynamics under climate change: long-term observations and model analysis. Oecologia 150(4):625-642

Tirok K, Gaedke U (2010) Internally driven alternation of functional traits in a multispecies predator-prey system. Ecology 91:17481762

Tirok K, Bauer B, Wirtz K, Gaedke U (2011) Predator-prey dynamics driven by feedback between functionally diverse trophic levels. PLoS ONE 6(11):e27,357. doi:10.1371/journal.pone.0027357. http://www.ncbi.nlm.nih.gov/pubmed/22096560 
Vasseur DA, Gaedke U (2007) Spectral analysis unmasks synchronous and compensatory dynamics in plankton communities. Ecology 88(8):2058-2071

Vergnon R, Dulvy NK, Freckleton RP (2009) Niches versus neutrality: uncovering the drivers of diversity in a species-rich community. Ecol lett 12(10):1079-1090
Webb CT, Hoeting JA, Ames GM, Pyne MI, n LeRoy P (2010) A structured and dynamic framework to advance traits-based theory and prediction in ecology. Ecol Lett 13:267-283

Wirtz KW, Eckhardt B (1996) Effective variables in ecosystem models with an application to phytoplankton succession. Ecol Model 92(1):33-53 